\title{
A Novel Transmitter Architecture for Spectrally-Precoded OFDM
}

\author{
Medhat Mohamad, Rickard Nilsson and Jaap van de Beek
}

\begin{abstract}
Frequency nulling spectral precoding is an approach that suppresses the out-of-band emission in OFDM systems. In this paper, we discuss the transmitter architecture of the spectrally precoded OFDM systems. We design a novel precoder that matches the practical implementation of the OFDM modulator. We show that spectral precoding can relax the analog low pass filtering requirements of the OFDM system transmitter. We examine the effect of spectral precoding on the PAPR as well as the effect of the PA on the spectral precoding suppression performance. We also study the compliance of the spectrally precoded OFDM transmitter with the 3GPP standardization measures and analyze its computation complexity. At the receiver side, we analyze the in-band interference and BER performance of the suggested precoding approach.
\end{abstract}

Index Terms-spectral precoding, OFDM, Out-of-Band emission, PAPR, discrete, analog.

\section{INTRODUCTION}

$\mathbf{T}$ HE 3GPP group has recently decided that the Cyclic Prefix Orthogonal Frequency Division Multiplexing (CPOFDM) will serve as the base waveform for the upcoming $5 \mathrm{G}$ systems operating in the sub- $6 \mathrm{GHz}$ band [1]. The attractive features of OFDM favored this decision as they have favored previously similar decisions for 4G [2]. Yet, OFDM has a couple of drawbacks that may limit its legitimation for some of the $5 \mathrm{G}$ systems.

Besides the high peak to average Power ratio (PAPR) another big drawback of OFDM is that the waveform exhibits high out-of-band (OOB) emission [3]. The OOB emission from the OFDM signal will cause interference on systems operating in neighboring bands. This OOB interference will degrade the bit error rate (BER) performance of neighboring systems. In 4G, 3GPP standards address the OOB emission problem by, firstly, reserving a guard interval between the different operators' frequency channels. The guard interval in $4 \mathrm{G}$ is $10 \%$ of the total dedicated bandwidth [4]. This waste of resources is not affordable for the spectral efficiency requirements of some of $5 \mathrm{G}$ systems operating within the sub$6 \mathrm{GHz}$ band.

Secondly, the 3GPP standards specify a measure, adjacent carrier leakage ratio (ACLR), as the ratio of the power transmitted within the OFDM channel bandwidth assigned to the vendor and the OOB emission power leaking into the adjacent channel [4]. Consequently, the LTE standards force

The authors are at the department of Computer Science, Electrical and Space Engineering, Luleå University of Technology, SE-97187, Luleå, Sweden. \{medhat.mohamad, rickard.o.nilsson, jaap.vandeBeek $\} @ 1 t u . s e$ the equipments to treat the OFDM signals so that the OOB emissions are suppressed below a certain ACLR.

The standards give vendors a freedom on the techniques they can use to suppress the OOB emission in OFDM systems. To assure that the practical OFDM signal is as close as possible to the reference OFDM signal, the LTE standards define another measure, the error vector magnitude (EVM), and require vendors to stick with specific EVM requirements [4]. EVM measures how close a practically implemented OFDM signal (measured directly after the front-end of the transmitter) is to the reference OFDM signal specified by the standards. Figure 1 shows the EVM measurement procedure.

Spectral precoding is a recent approach that suppresses the OOB emission in OFDM by manipulating the correlation property between the data symbols modulating the OFDM subcarriers. The spectral precoding approach introduced in [5], develops new basis signals that achieve high spectral compactness. The new basis signals are designed from the rectangular basis set by manipulating the correlation property of the modulating data symbols. The designed basis set has continuous phase characteristics. In other words, the new basis signals have zero interval edges. Consequently, the whole OFDM symbol constructed from these basis subcarriers decays to zero at the edges.

Another approach that focuses on smoothing the OFDM signal to improve the spectral compactness is introduced in [6]-[8]. While [5] focus on designing new basis signals, [6][8] directly deal with the discontinuity property of the OFDM signal and force the successive OFDM symbols continuous by precoding the data symbols.

The precoding approaches discussed above improve the OFDM spectral efficiency by changing the OFDM signal's characteristics in the time domain (precode the OFDM signal to become smooth and continuous). Another approach that shows high flexibility and efficiency in suppressing the OOB emission is the frequency nulling approach discussed in [9]. Contrary to the spectral precoding approaches we discussed above, the frequency nulling approach improves the OFDM spectral efficiency by looking at the OFDM signal in the frequency domain rather than in the time domain. In the frequency nulling approach, the OOB emission in OFDM is suppressed by introducing a set of nulls at well chosen frequencies in the spectrum. The frequency nulls make the whole spectrum decays faster than $\frac{1}{f^{2}}$. The number of nulls, as well as the frequencies of these nulls, controls the level of the OOB emission suppression. This approach outperforms the 


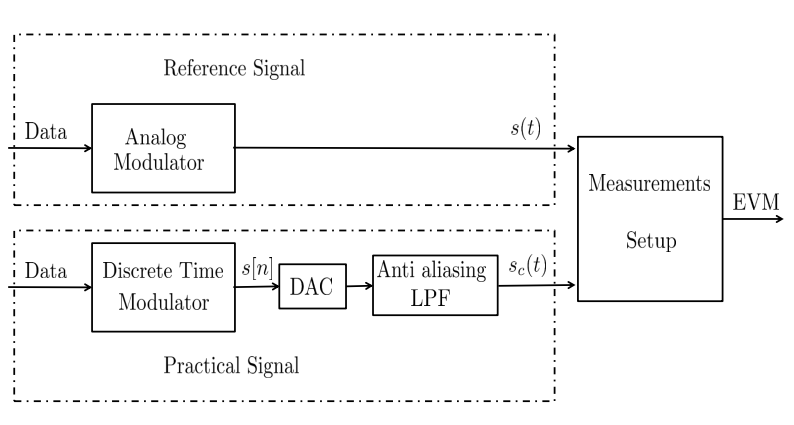

Fig. 1. Reference versus practical implementation of the OFDM system. EVM is tested directly at the output of the transmitter. EVM measures how close the practical signal resembles the reference signal.

other precoding approaches in its spectral flexibility which is controlled by the choice of the nulls. This spectral flexibility is favorable in cognitive radio systems where communications systems need to adaptively change their spectral characteristics.

The different spectral precoders introduced in [6]-[12] and others assume that the original plain OFDM signal to be precoded is the reference OFDM signal specified by the LTE standards [4] as well as DVB, DAB, WiFi, ...etc. This reference OFDM signal resembles the historical OFDM signal presented in the famous paper of 1966 [13]. Yet, we can only implement the reference OFDM signal using analog setup. However, without exception, practical systems, nowadays, construct the continuous OFDM signal with a digital setup [14]. Although the exception precoding approaches in [15] and [16] assume that the original OFDM signal is the discrete signal, yet the papers don't study the precoded OFDM transmitter architecture. So far, no contributions have addressed the transmitter implementation architecture of the spectrally precoded OFDM systems.

As a consequence of the practical implementation, the OFDM modulator mismatches the different spectral precoders suggested in [6]-[12]. Incidentally, [6]-[12] mimic the analog reference OFDM signal by extreme interpolation of the discrete-time OFDM signal. We can interpolate the discretetime signal by choosing a large inverse discrete Fourier transform (IDFT) size compared to the number of subcarriers. Yet, exaggerating the IDFT block size is impractical for real time OFDM implementation.

Here, rather than interpolating the discrete-time OFDM signal to mimic the continuous OFDM reference signal, we, in this paper, implement a practical discrete-time precoder that fits the typical discrete-time implementations of the OFDM signal. A new precoder and transmitter architecture emerges whose performance we study with the LTE measures introduced above. In this paper, in contrast to precoded OFDM signals, we will refer to a signal without spectral precoding as a plain OFDM signal. Similarly, we will refer to the continuous time standardized OFDM signal as a reference signal in contrast to vendor specific signals which we refer to as practical signals. In Section II we introduce the practical model of the plain OFDM signal and compare it with the ref-

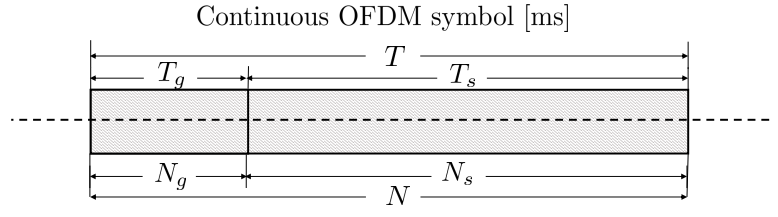

Discrete OFDM symbol [samples]

Fig. 2. A scheme of a continuous OFDM symbol versus a discrete OFDM symbol.

erence model of the plain OFDM signal. Then, in Section III, we discuss frequency nulling spectral precoding techniques. In III-A We discuss the spectral precoding for the reference signal. In III-B we introduce a precoder matched to the practical OFDM model and we analyze its OOB emission suppression. Furthermore, we study the influence of the anti-aliasing LPF of the frontend electronics on the precoded OFDM spectra. In Section IV we study the influence of the spectral precoding on the PAPR of the OFDM signals. We also examine the effect of the power amplifier (PA) signal's nonlinearities on the OOB emission suppression performance. In Section V, we analyze the performance of the precoded OFDM system at the transmitter side of the communications link as well as at the receiver side and we examine the compliance of spectral precoding with LTE requirements. Finally, we conclude our work in section VI. For clarity, Table IV at the end of this paper collects the different symbols used in the paper.

\section{IDEAL VERSUS PRACTICAL MODEL OF OFDM}

In essence, LTE defines the reference OFDM signal, $s(t)$, by [2]

$$
s(t)=\sum_{i=-\infty}^{+\infty} s_{i}(t-i T)
$$

where $T=T_{s}+T_{g}, T_{g}$ is the length of the cyclic prefix and $T_{s}$ is the length of the OFDM symbol and

$$
s_{i}(t)=\sum_{k \in \mathcal{K}} \bar{d}_{k, i} e^{-j 2 \pi \frac{k}{T_{s}} t} I(t),
$$

where $\bar{d}_{k, i}$ is the $k^{\text {th }}$ data symbol modulating the $k^{\text {th }}$ subcarrier and $k \in \mathcal{K}=\left\{k_{0}, k_{1}, \ldots, k_{K-1}\right\}$. If $\bar{d}_{k, i}=d_{k, i}$, then $s(t)$ is a plain OFDM signal (without spectral precoding) and $d_{k, i}$ belongs to some constellation set $\mathbb{C}$. The indicator function $I(t)=1$ for $-T_{g} \leq t \leq T_{s}$ and $I(t)=0$ elsewhere. Figure 2 (Top) shows a scheme of a continuous OFDM symbol.

The Fourier transform of the symbol, $s_{i}(t)$, is

$$
\begin{aligned}
\tilde{S}_{i}(f) & =\mathcal{F}\left\{s_{i}(t)\right\}=\mathcal{F}\left\{\sum_{k \in \mathcal{K}} \bar{d}_{k, i} e^{-j 2 \pi \frac{k}{T_{s}} t} I(t)\right\} \\
& =\sum_{k \in \mathcal{K}} \bar{d}_{k, i} \mathcal{F}\left\{e^{\frac{-j 2 \pi k t}{T_{s}}} I(t)\right\}=\sum_{k \in \mathcal{K}} \bar{d}_{k, i} \tilde{a}_{k}(f),
\end{aligned}
$$

where $\tilde{a}_{k}(f)$ is a shifted version of the sinc function that represents the Fourier transform of the $k^{\text {th }}$ rectangularly windowed continuous complex exponential subcarrier,

$$
\tilde{a}_{k}(f)=T e^{-j \pi\left(T_{s}-T_{g}\right)\left(f-\frac{k}{T_{s}}\right)} \operatorname{sinc}\left(\pi T\left(f-\frac{k}{T_{s}}\right)\right),
$$


where $\operatorname{sinc}(x)=\frac{\sin (x)}{\tilde{\tilde{S}}}$ is the cardinal sine. Using vector notation we rewrite $\tilde{S}_{i}(f)$ as

$$
\tilde{S}_{i}(f)=\tilde{\mathbf{a}}^{T}(f) \overline{\mathbf{d}}_{i},
$$

For the reference plain OFDM signal, $s(t), \quad \tilde{\mathbf{a}}(f) \triangleq$ $\left[\tilde{a}_{k_{0}}(f), \tilde{a}_{k_{1}}(f), \ldots, \tilde{a}_{k_{K-1}}(f)\right]^{T}$ and $\overline{\mathbf{d}}_{i}=\mathbf{d}_{i}$ such that $\overline{\mathbf{d}}_{i} \triangleq$ $\left[\bar{d}_{k_{0}}, \bar{d}_{k_{1}}, \ldots, \bar{d}_{k_{K-1}}\right]^{T}$ and $\mathbf{d}_{i} \triangleq\left[d_{k_{0}}, d_{k_{1}}, \ldots, d_{k_{K-1}}\right]^{T}$ where $[.]^{T}$ stands for the Matrix (or vector) transpose.

From (5), and assuming that the original data symbols are uncorrelated, we can compute the power spectrum of the reference OFDM signal as [9]

$$
\begin{aligned}
\tilde{P}(f) & =\frac{1}{T} E\left\{\left|\tilde{S}_{i}(f)\right|^{2}\right\}=\frac{1}{T} \tilde{\mathbf{a}}^{T}(f) E\left\{\mathbf{d}_{i} \mathbf{d}_{i}{ }^{H}\right\} \tilde{\mathbf{a}}^{*}(f) \\
& =\frac{1}{T} \tilde{\mathbf{a}}^{T}(f) \tilde{\mathbf{a}}^{*}(f),
\end{aligned}
$$

where $*$ is the complex conjugate.

Clearly we can distinctly implement $s(t)$ in (1) using the analog circuit as presented in Figure 1. In the analog modulator each data symbol modulates a continuous time rectangularly windowed complex sinusoid with finite duration, $T_{s}$, and center frequency $f_{k}=\frac{k}{T_{s}}$. We would generate the continuous OFDM symbol by adding the modulated sinusoids together. Notably, the LTE reference signal (1), was the OFDM embodiment as described in the landmark paper [13] in 1966 when digital circuitry was still in its infancy.

However, the analog implementation of the reference OFDM signal, $s(t)$, is impractically costly as we would require $K$ electronic mixers to modulate $K$ subcarriers with $K$ data symbols. Moreover, the mixing process of each modulation branch would need to operate accurately at $f_{k}$. Otherwise, the orthogonality between the different subcarriers is violated. This operating accuracy means that we require higher cost mixers.

These drawbacks of the analog implementation of the OFDM system kept OFDM under the shadow for years. It is not until the maturity of the digital electronics technologies in 1990s when [14] proposed to move toward the discretetime implementation of the OFDM signal. The proposal in [14] used the discrete Fourier transform process (DFT) for the implementation of the practical OFDM system.

Today's state-of-art transmitters are better modeled with a practical discrete-time OFDM signal [17]

$$
s[n]=\sum_{i=-\infty}^{+\infty} s_{i}[n-i N],
$$

where $s_{i}[n]$ is the $i^{\text {th }}$ discrete-time OFDM symbol of length $N=N_{s}+N_{g}$ samples. $N_{g}$ is the number of samples required by the CP extension and $N_{s}$ is the number of samples in the OFDM symbol. Generation of $s_{i}[n]$, is simply an IDFT process such that

$$
s_{i}[n]=\sum_{k \in \mathcal{K}} \bar{d}_{k, i} e^{-2 j \pi \frac{k}{N_{s}} n} I[n],
$$

Where in (8), $N_{s}$ also represents the size of the IDFT block and the indicator function, $I[n]=1$ for $-N_{g} \leq n \leq N_{s}$ and
$I[n]=0$ elsewhere. Figure 2 (bottom) shows a scheme of a discrete OFDM symbol.

The discrete-time Fourier transform of $s_{i}[n]$ is

$$
\begin{aligned}
S_{i}(\omega) & =\mathcal{F}\left\{s_{i}[n]\right\}=\mathcal{F}\left\{\sum_{k \in \mathcal{K}} \bar{d}_{k, i} e^{\frac{-j 2 \pi k}{N_{s}} n} I[n]\right\} \\
& =\sum_{k \in \mathcal{K}} \bar{d}_{k, i} \mathcal{F}\left\{e^{\frac{-j 2 \pi k}{N_{s}} n} I[n]\right\}=\sum_{k \in \mathcal{K}} \bar{d}_{k, i} a_{k}(\omega) .
\end{aligned}
$$

where

$$
a_{k}(\omega)=e^{-j \frac{N_{s}-N_{g}}{2}\left(\omega-\frac{2 \pi k}{N_{s}}\right)} \frac{\sin \left((N+0.5)\left(\omega-\frac{2 \pi k}{N_{s}}\right)\right)}{\sin \left(\frac{\omega-\frac{2 \pi k}{N_{s}}}{2}\right)},
$$

and $\omega \in[0,2 \pi]$ is the normalized frequency and $a_{k}(\omega)$ is a time shifted version of the Dirichlet function. Similar to (5),

$$
S_{i}(\omega)=\mathbf{a}(\omega)^{T} \overline{\mathbf{d}}_{i}
$$

where, here, $\mathbf{a}(\omega) \triangleq\left[a_{k_{0}}(\omega), a_{k_{1}}(\omega), \ldots, a_{k_{K-1}}(\omega)\right]$. From (11), the power spectrum $P(\omega)$ of the discrete-time OFDM signal becomes

$$
P(\omega)=\frac{1}{N} E\left\{\left|S_{i}(\omega)\right|^{2}\right\}=\frac{1}{N} \mathbf{a}^{T}(\omega) E\left\{\mathbf{d}_{i} \mathbf{d}_{i}{ }^{H}\right\} \mathbf{a}^{*}(\omega) .
$$

Assuming that the original data symbols are uncorrelated, this implies that

$$
P(\omega)=\frac{1}{N} \mathbf{a}^{T}(\omega) \mathbf{a}^{*}(\omega) .
$$

Practical communications transmitters convert the discrete OFDM signal to a continuous signal, by using a dual DAC followed by an anti-imaging filter [18]. The DAC can be either a pulse width modulation (PWM) DAC, or $\triangle \Sigma$ DAC.

Figure 4 shows the DAC followed by the anti-imaging LPF. We can mathematically model the DAC conversion into two stages [19]. Of course those two mathematical stages are implicit in the practical implementation of the DAC (i.e. they take place jointly through the electronic components of the DAC). In the first stage, the DAC multiplies the discrete-time OFDM signal sequence, $s[n]$, with an impulse train such that

$$
x(t)=\sum_{n=-\infty}^{+\infty} s[n] \delta\left(t-\frac{n}{f_{\mathrm{cl}}}\right),
$$

where $\delta(t)$ denotes delta dirac and $f_{\mathrm{cl}}$ is the construction clock frequency of the DAC such that $f_{\mathrm{cl}}=\frac{N_{s}}{T_{s}}$.

The power spectrum of the continuous-time OFDM signal, $x(t)$, constructed from the discrete-time signal is

$$
P^{x}(f)=\frac{1}{T} \mathbf{a}^{T}(f) \mathbf{a}^{*}(f),
$$

where the frequency index, $f$, is related to the normalized frequency $\omega$ by

$$
f=\frac{\omega L}{2 \pi} \cdot f_{\mathrm{cl}},
$$

and $L \in \mathbb{Z}-\{0\}$. Note that, $P^{x}(f)$ is constituted of copies of the spectrum in the $\left[0, f_{\mathrm{cl}}\right]$ band. This means that the construction clock frequency controls how close the spectrum aliases 

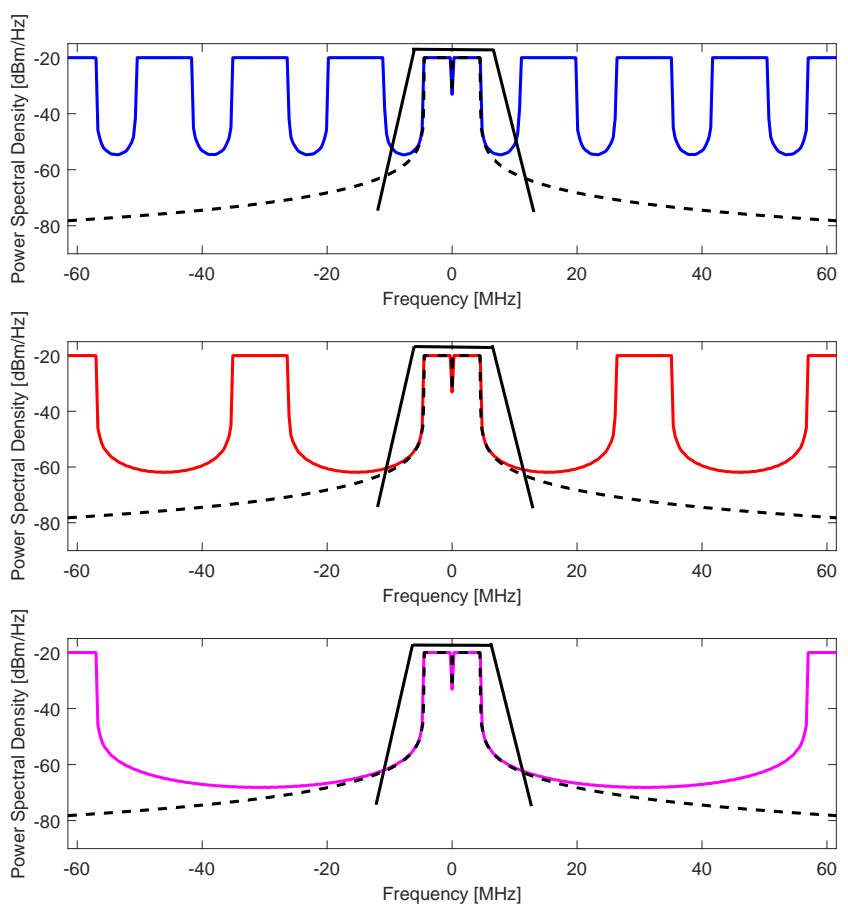

Fig. 3. Spectra of three continuous time plain OFDM signals. We constructed the three signals from three discrete-time signals with construction clock frequencies: $f_{\mathrm{cl}}=\frac{1024}{T_{\mathrm{s}}} \mathrm{Hz}$ (top), $f_{\mathrm{cl}}=\frac{2048}{T_{\mathrm{s}}} \mathrm{Hz}$ (middle) and $f_{\mathrm{cl}}=\frac{4096}{T}$ Hz. We compare the three spectra to the spectrum of the reference OFDM signal (black dashed). As the DAC construction clock frequency increases the practical signal spectrum approaches the reference signal spectrum.The trapezoidal solid line represents the frequency response of the analog antiimaging LPF.

are to each other. Figure 3 shows the spectrum $P^{x}(f)$ for three different construction clock frequencies: $f_{\mathrm{cl}}=\frac{1024}{T_{\mathrm{s}}}=15.36$ $\mathrm{MHz}, f_{\mathrm{cl}}=\frac{2048}{T_{\mathrm{s}}}=30.72 \mathrm{MHz}$ and $f_{\mathrm{cl}}=\frac{4096}{T_{\mathrm{s}}}=61.44 \mathrm{MHz}$. Here we choose $T_{s}=\frac{1}{15} \mathrm{~ms}$ equal to the OFDM symbol time used in LTE systems. We compare the three spectra at those three construction clock frequencies with the spectrum of the reference OFDM signal, $\tilde{P}(f)$. As the construction clock frequency increases, the spectrum, $P^{x}(f)$, becomes closer to the spectrum, $\tilde{P}(f)$, of the reference OFDM signal, $s(t)$. As $f_{\text {cl }}$ goes to infinity $P^{x}(f)$ matches exactly $\tilde{P}(f)$.

The second conceptual stage of the DAC constructs the continuous signal from the pulse train. This stage is mathematically equivalent to filtering the pulse train with a reconstruction LPF. The reconstruction stage of the DAC is typically too weak to completely filter out the aliases as Figure 4 shows. Therefore, an anti aliasing filter usually follows the DAC [18]. The signal output of the anti-imaging filter becomes

$$
s_{c}(t)=x(t) * h(t),
$$

where $h(t)$ is the joint impulse response of the DAC filter and the anti-imaging LPF. The power spectrum of the practical filtered continuous OFDM signal, $s_{c}(t)$, is

$$
P(f)=|H(f)|^{2} P^{x}(f),
$$

where $H(f)$ is the joint frequency response of the DAC reconstruction filter and the anti-imaging LPF.

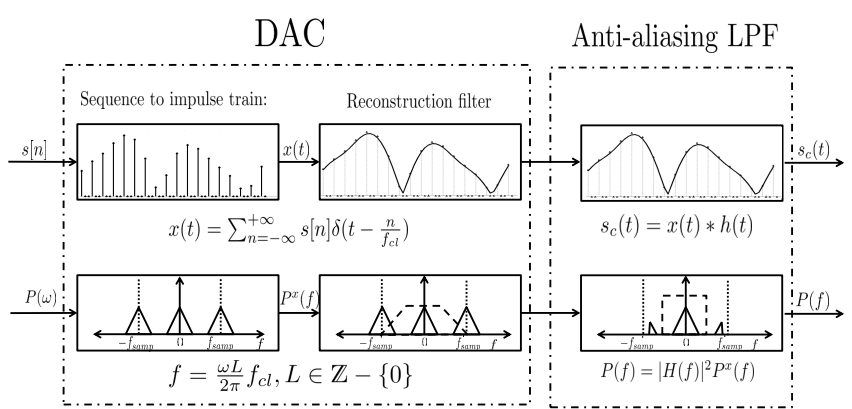

Fig. 4. A mathematical model of the DAC followed by an anti-imaging LPF. The model shows the behavior of the processed signal in time domain (upper figure) as well as the spectrum of the signal (lower figure).

\section{Frequency Nulling Spectral Precoding}

Figure 3 shows that the spectrum of the plain OFDM signal possesses high OOB emission. At its best, the plain OFDM spectrum decays as a factor of $\frac{1}{f^{2}}$. Classical practical OFDM systems suppress the OOB emission in OFDM by digital low pass filtering of the plain OFDM signal. A digital LPF filters the baseband discrete-time OFDM signal before the DAC process [20], [21]. The use of a digital LPF for suppressing the $\mathrm{OOB}$ emission typically relaxes the design requirements of the anti-imaging LPF. Alternatively, the frequency nulling precoder suggested in [9], can suppress OOB emission and, therefore, replaces the digital LPF [22].

\section{A. Reference spectrally precoded OFDM}

Frequency nulling spectral precoding nulls the spectrum at a set of $M$ adequately chosen frequencies, $\left\{f_{0}, f_{1}, \ldots, f_{M-1}\right\}$. Nulling the spectrum at these frequencies forces the whole spectrum to decay faster than $\frac{1}{f^{2}}$, see [9]. To generate the nulls in the spectrum, the precoder, $\tilde{\mathbf{G}}$, linearly combines the original data symbols collected in $\mathbf{d}_{i}$ such that

$$
\overline{\mathbf{d}}_{i}=\tilde{\mathbf{G}} \mathbf{d}_{i} .
$$

As a consequence, the OFDM system modulates the subcarriers with $\overline{\mathbf{d}}_{i}$ rather than $\mathbf{d}_{i}$. The precoder generates $\overline{\mathbf{d}}_{i}$ so that the spectrum is notched at the specified frequencies. This means that $\overline{\mathbf{d}}_{i}$ satisfies

$$
\tilde{\mathbf{a}}^{T}\left(f_{m}\right) \overline{\mathbf{d}}_{i}=0,
$$

where $\tilde{\mathbf{a}}\left(f_{m}\right) \triangleq\left[\tilde{a}_{k_{0}}\left(f_{m}\right), \tilde{a}_{k_{1}}\left(f_{m}\right), \ldots \tilde{a}_{k_{K-1}}\left(f_{m}\right)\right]^{T}$ is a vector collecting the values of the subcarriers at the nulling frequency $f_{m}$. If we define the constraint matrix $\tilde{\mathbf{A}} \triangleq$ $\left[\tilde{\mathbf{a}}\left(f_{0}\right), \tilde{\mathbf{a}}\left(f_{1}\right), \ldots, \tilde{\mathbf{a}}\left(f_{M-1}\right)\right]^{T}$ as an $M \times K$ matrix that collects the values of the $K$ subcarriers at the $M$ nulling frequencies, then the precoder, $\tilde{\mathbf{G}}$, requires that

$$
\tilde{\mathbf{A}} \overline{\mathbf{d}}_{i}=\mathbf{0} \text {. }
$$

In words $\overline{\mathbf{d}}_{i}$ should fall in the null space $\mathcal{N}(\tilde{\mathbf{A}})$ of $\tilde{\mathbf{A}}$ and therefore, the precoder $\tilde{\mathbf{G}}$, is the complimentary projection matrix such that

$$
\tilde{\mathbf{G}}=\mathbf{I}-\tilde{\mathbf{A}}^{H}\left(\tilde{\mathbf{A}} \tilde{\mathbf{A}}^{H}\right)^{-1} \tilde{\mathbf{A}} .
$$




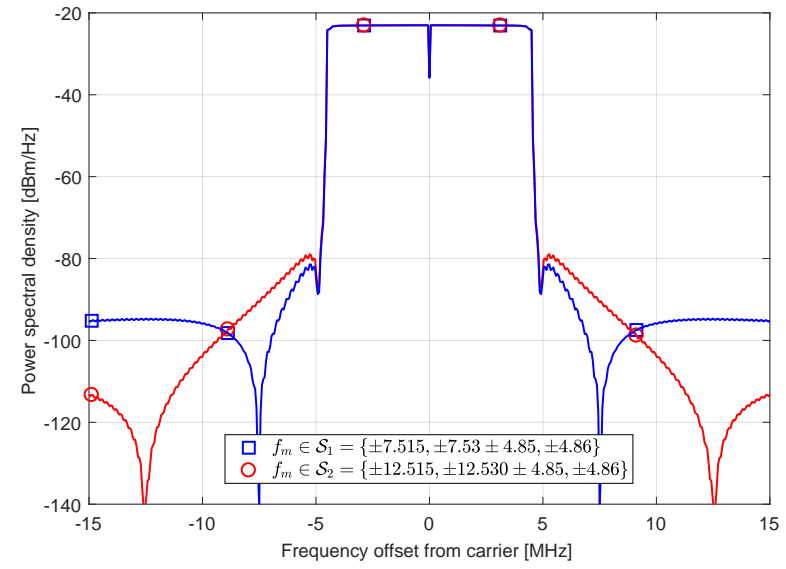

Fig. 5. Spectra of two reference OFDM signals precoded by $\tilde{\mathbf{G}}$, defined in (22). The figure shows the dependency of the OOB emission suppression on the location of the nulls.

Strictly speaking, the fundamental concept of the precoder is to confine the original data vector $\mathbf{d}_{i}$ into a specific subspace before the DFT modulation of the OFDM transmitter. The subspace is determined a priori to guarantee a set of nulls in the OFDM spectrum. The introduced nulls force the whole spectrum to decay fast.

Then, similar to (6), the spectrum of the reference precoded OFDM signal becomes

$$
\begin{aligned}
\tilde{P}_{p}(f) & =\frac{1}{T} E\left\{\left|\tilde{S}_{i}(f)\right|^{2}\right\}=\frac{1}{T} \tilde{\mathbf{a}}^{T}(f) E\left\{\overline{\mathbf{d}}_{i} \overline{\mathbf{d}}_{i}^{H}\right\} \tilde{\mathbf{a}}^{*}(f) \\
& =\frac{1}{T} \tilde{\mathbf{a}}^{T}(f) \tilde{\mathbf{G}} E\left\{\mathbf{d}_{i} \mathbf{d}_{i}^{H}\right\} \tilde{\mathbf{G}}^{H} \tilde{\mathbf{a}}^{*}(f) . \\
& =\frac{1}{T} \tilde{\mathbf{a}}^{T}(f) \tilde{\mathbf{G}} \tilde{\mathbf{a}}^{*}(f) .
\end{aligned}
$$

Under the consideration that the original data symbols in $\mathbf{d}_{i}$ are uncorrelated i.e. $E\left\{\mathbf{d}_{i} \mathbf{d}_{i}^{H}\right\}=\mathbf{I}$, (23) collapses to

$$
\tilde{P}_{p}(f)=\frac{1}{T} \tilde{\mathbf{a}}^{T}(f) \tilde{\mathbf{G}} \tilde{\mathbf{G}}^{H} \tilde{\mathbf{a}}^{*}(f) .
$$

Using the Idempotency and the Hermitian properties of the projection matrix, $\tilde{\mathbf{G}}$, we can rewrite (24) as

$$
\tilde{P}_{p}(f)=\frac{1}{T} \tilde{\mathbf{a}}^{T}(f) \tilde{\mathbf{G}} \tilde{\mathbf{a}}^{*}(f) .
$$

Note that the capability of the precoder to suppress the OOB emission is dependent on the number of nulling frequencies, $f_{m}$, the precoder introduces as well as their locations. As the number of nulls increases the suppression performance improves. On the other hand, the location of the nulls in spectral precoding is still a matter of research. Two important observations regarding the location of the nulls are relevant here: Firstly, for the nulls to be able to suppress the whole spectrum we should pair at least two nulls adjacent to each other [9]. Secondly the suppression of the emissions decays as we move the pair of nulls away from each other. This means that the spectral band between the pairs of nulls suffers less OOB emission as we bring the pairs closer to each other. Figure 5 illustrates those two observations. The figure shows the spectra of two reference OFDM signals precoded with $\tilde{\mathbf{G}}$.

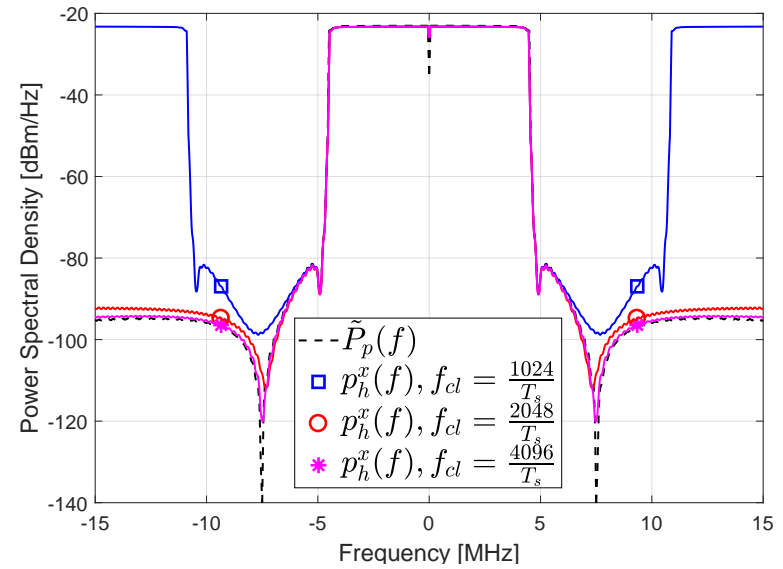

Fig. 6. Spectra of three precoded practical OFDM signals with construction clock frequencies: $\frac{1024}{T_{s}}=15.36 \mathrm{MHz}, \frac{2048}{T_{s}}=30.72 \mathrm{MHz}$ and $\frac{4096}{T_{s}}=61.44 \mathrm{MHz}$. The nulls are at $f_{m} \in \mathcal{S}_{1}$. We compare the three spectra to the spectrum of a precoded reference OFDM signal (black dashed). As the construction clock frequency decreases the spectrum of the precoded practical OFDM signal drifts away from the spectrum of the precoded reference OFDM signal. Consequently, the OOB emission suppression capability of the mismatched precoder decreases.

In the first precoded spectrum we designed $\tilde{\mathbf{G}}$ to introduce eight nulls at $f_{m} \in \mathcal{S}_{1}=\{ \pm 7.515, \pm 7.530 \pm 4.85, \pm 4.86\}$ MHz. While in the second precoded spectrum, we designed $\mathbf{G}$ to introduce eight nulls at $f_{m} \in \mathcal{S}_{2}=\{ \pm 12.515, \pm 12.350 \pm$ $4.85, \pm 4.86\} \mathrm{MHz}$.

\section{B. Practical spectrally precoded OFDM}

Since [9] designs the precoder based on the reference model of the OFDM system, $\tilde{\mathbf{G}}$ only matches OFDM systems implemented using the analog setup. Yet, as we mentioned in Section II that the analog implementation of the OFDM system is irrelevant in today's transmitters. Applying the precoder, $\tilde{\mathbf{G}}$, presented in [9] over practical OFDM systems needs a careful and critical assessment as it will cause a mismatch: The precoder in [9] is not designed to operate in conjunction with practical OFDM modulators.

The spectrum of the mismatched precoded OFDM signal becomes

$$
P_{h}^{x}(f)=\frac{1}{T} \mathbf{a}^{T}(f) \tilde{\mathbf{G}} \mathbf{a}^{*}(f) .
$$

In (26), $P_{h}^{x}(f)$ is the precoded spectrum at the output of the first stage of the DAC. We can say that $P_{h}^{x}(f)$ is the mismatched precoded version of $P^{x}(f)$. The assumption in [9] holds if the construction clock frequency is high enough so that the spectrum of the discrete-time OFDM signal is close enough to the spectrum of the reference OFDM signal. Although for the practical OFDM signals the construction clock is limited by the complexity and the implementation requirements of the system. Therefore the spectrum of the practical implemented OFDM signal (both plain or precoded) can never match the spectrum of the reference OFDM signal. As a consequence, the precoder suggested in [9], mismatches the OFDM system. Although, similar to our discussion in Section II, the practical precoded spectrum $P_{h}^{x}(f)$ in (26) approaches the precoded 
TABLE I

SPECTRA OF PLAIN, MATCHED PRECODED, MISMATCHED PRECODED REFERENCE VERSUS PRACTICAL OFDM SIGNALS.

\begin{tabular}{|c|c|c|c|}
\hline & Reference OFDM signal & Output of the DAC:stage I & Output of the anti-imaging LPF \\
\hline $\begin{array}{c}\text { Plain } \\
\text { OFDM signal }\end{array}$ & $\tilde{P}(f)=\frac{1}{T} \tilde{\mathbf{a}}^{T}(f) \tilde{\mathbf{a}}^{*}(f)$ & $P^{x}(f)=\frac{1}{T} \mathbf{a}^{T}(f) \mathbf{a}^{*}(f)$ & $P(f)=\frac{1}{T}|H(f)|^{2} \mathbf{a}^{T}(f) \mathbf{a}^{*}(f)$ \\
\hline $\begin{array}{c}\text { Precoded OFDM signal } \\
\text { using the precoder in [9] }\end{array}$ & $\tilde{P}_{p}(f)=\frac{1}{T} \tilde{\mathbf{a}}^{T}(f) \tilde{\mathbf{G}} \tilde{\mathbf{a}}^{*}(f)$ & $P_{h}^{x}(f)=\frac{1}{T} \mathbf{a}^{T}(f) \tilde{\mathbf{G}} \mathbf{a}^{*}(f)$ & $P_{h}(f)=\frac{1}{T}|H(f)|^{2} \mathbf{a}^{T}(f) \tilde{\mathbf{G}} \mathbf{a}^{*}(f)$ \\
\hline $\begin{array}{c}\text { Precoded OFDM signal } \\
\text { using the practical precoder }\end{array}$ & N.A. & $P_{p}^{x}(f)=\frac{1}{T} \mathbf{a}^{T}(f) \mathbf{G} \mathbf{a}^{*}(f)$ & $P_{p}(f)=\frac{1}{T}|H(f)|^{2} \mathbf{a}^{T}(f) \mathbf{G a}^{*}(f)$ \\
\hline
\end{tabular}

reference spectrum $\tilde{P}_{p}(f)$ in (25) as the construction clock frequency increases.

Figure 6 shows the spectra of precoded practical OFDM signals analyzed just before the reconstruction LPF of the DAC. The OFDM system uses clock frequencies similar to those in Figure 3. The figure compares the practical precoded OFDM signal spectrum to the spectrum of a precoded reference OFDM signal. We precode the two signals with $\tilde{\mathbf{G}}$ which introduces 8 nulls at $f_{m} \in \mathcal{S}_{1}$.

From the analysis in Figure 6 we conclude that as the clock frequency decreases, the mismatch increases and, therefore, the OOB emission suppression performance deteriorates.

To overcome this problem, rather than using high construction frequency in the practical OFDM signal to match the reference model precoder, in this paper, we redesign a discretetime precoder

$$
\mathbf{G}=\mathbf{I}-\mathbf{A}^{H}\left(\mathbf{A} \mathbf{A}^{H}\right)^{-1} \mathbf{A} .
$$

The precoder, $\mathbf{G}$, introduces the nulls in the spectrum of the discrete-time signal model contrary to $\tilde{\mathbf{G}}$ that introduces the nulls upon the spectrum of the continuous time signal model (1), i.e. $\mathbf{G}$ focuses on the spectrum of the OFDM signal before being constructed by the DAC. For this discrete-time precoder we define the constraint matrix $\mathbf{A}$ of size $M \times K$ as $\mathbf{A} \triangleq\left[\mathbf{a}\left(\omega_{0}\right), \mathbf{a}\left(\omega_{1}\right), \ldots, \mathbf{a}\left(\omega_{M-1}\right)\right]$. Where $\mathbf{a}\left(\omega_{m}\right)$ is a vector collecting the values of the $K$ subcarriers at the normalized nulling frequency $\omega_{m}$ i.e. for the discrete-time precoder we substitute $\omega$ in (10) with $\omega_{m}$ such that

$$
a_{k}\left(\omega_{m}\right)=e^{-j \frac{N_{s}-N_{g}}{2}\left(\omega_{m}-\frac{2 \pi k}{N_{s}}\right)} \cdot \frac{\sin \left((N+0.5)\left(\omega_{m}-\frac{2 \pi k}{N_{s}}\right)\right)}{\sin \left(\frac{\omega_{m}-\frac{2 \pi k}{N_{s}}}{2}\right)} .
$$

In words we evaluate the Dirichlet subcarriers of the discrete OFDM signal at the normalized nulling frequency $\omega_{m}$. Then, as in (27), the spectrum of the discrete OFDM signal precoded by $\mathbf{G}$ becomes

$$
\begin{aligned}
P_{p}(\omega) & =\frac{1}{N} E\left\{\left|S_{i}(\omega)\right|^{2}\right\}=\frac{1}{N} \mathbf{a}^{T}(\omega) E\left\{\overline{\mathbf{d}}_{i} \overline{\mathbf{d}}_{i}^{H}\right\} \mathbf{a}^{*}(\omega) \\
& =\frac{1}{N} \mathbf{a}^{T}(\omega) \mathbf{G a}^{*}(\omega),
\end{aligned}
$$

where

$$
\overline{\mathbf{d}}_{i}=\mathbf{G d}_{i}
$$

With this precoder, the first processing stage of the DAC constructs the continuous time precoded signal that has the spectrum

$$
P_{p}^{x}(f)=\frac{1}{T} \mathbf{a}^{T}(f) \mathbf{G a}^{*}(f),
$$

where $f$ is related to $\omega$ as in (16) (in contrast to the spectrum of the mismatched precoder $P_{h}^{x}(f)$ in (26)).

Figure 7 compares $P_{p}^{x}(f)$ with $P_{h}^{x}(f)$. The figure also shows the spectrum $\tilde{P}_{p}(f)$. The figure shows how much more suppression we can accomplish if we match the spectral precoder to the discrete-time modulator. The suppression performance of a properly matched precoder is better than the suppression performance of the precoder designed for the reference signal. In Figure 7 , the DAC operates at a construction clock frequency $f_{\mathrm{cl}}=\frac{1024}{T s}$.

After the DAC constructs the precoded OFDM signal, the anti-imaging LPF filters the precoded practical OFDM signal so that

$$
P_{h}(f)=|H(f)|^{2} P_{h}^{x}(f)
$$

and

$$
P_{p}(f)=|H(f)|^{2} P_{p}^{x}(f),
$$

where $P_{h}(f)$ and $P_{p}(f)$ are the spectra of the filtered OFDM signal when we use a mismatched precoder, $\tilde{\mathbf{G}}$ in (32), versus a matched precoder, $\mathbf{G}$ in (33), respectively. Figure 8 shows the two filtered spectra, $P_{h}(f)$ and $P_{p}(f)$, compared to the spectrum of a filtered plain practical OFDM signal, $P(f)$. The figure uses the same DAC used to evaluate Figure 7.

The anti-imaging filter we used in Figure 8 is a Chebyshev type II filter ${ }^{1}$. We choose the Chebyshev II filter due to its performance superiority in OFDM systems over the other types of filters [21]. The filter is of $10^{\text {th }}$ order and has a cutoff frequency $f_{c}=450 \cdot 15 \cdot 10^{3}=6.75 \mathrm{MHz}$ (i.e. we design the filter with a cutoff frequency equal to the bandwidth of an LTE based OFDM system with 900 subcarriers operating mode) and $-80 \mathrm{~dB}$ stop band ripples. These design characteristics of the anti-imaging LPF controls its complexity. The higher the filter order and the deeper the bandstop ripples are the more efficient the LPF but, on the other hand, the more complex to implement. Figure 9 shows the spectra, $P_{h}(f)$ and $P_{p}(f)$,

\footnotetext{
${ }^{1}$ To plot Figures 8 and 9, we mimic the analog Chebyshev II LPF with a digital Chebyshev II LPF using Matlab signal processing toolbox. Matlab transforms the analog LPF into digital LPF using the bilinear transformation. Check Matlab documentation and [19] page 538 for details.
} 


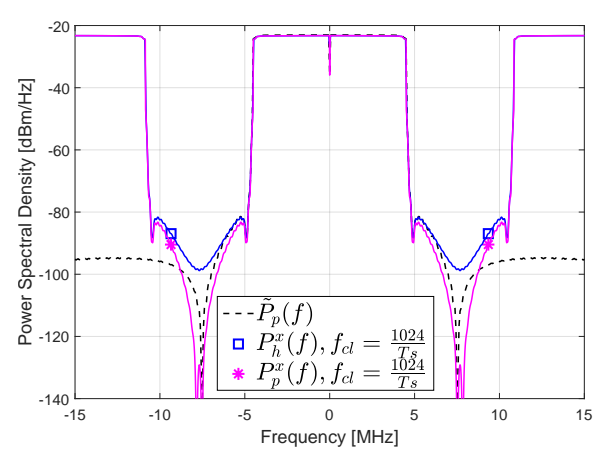

Fig. 7. Spectrum of a practical OFDM signal precoded with a mismatched precoder, $P_{h}^{x}(f)$, versus the spectrum of a practical OFDM signal precoded with a matched precoder, $P_{p}^{x}(f)$, compared to the spectrum of a precoded reference OFDM signal, $\tilde{P}_{p}(f)$. the DAC operates at a construction clock frequency $f_{\mathrm{cl}}=\frac{1024}{T s}$.

compared to the spectrum $P(f)$. The figure uses the same DAC used in figures 7 and 8 but a Chebyshev type II filter with a filter order of 7 rather than 10. In section $\mathrm{V}$ we show that Spectral precoding (especially using the matched precoder) can noticeably relax the requirements of the analog antialiasing LPF which consequently decreases the LPF design complexity.

Table I summarizes the different spectra of the two OFDM signal models we discuss in this paper.

\section{DYNAMIC RANGE CHARACTERISTICS OF PLAIN VERSUS PRECODED OFDM TRANSMITTERS}

Generally, the OFDM signal (both plain and precoded) is extremely sensitive to the analog front-end electronics [23]. The front-end setup may cause a severe deformation in the shape of the anti-imaging filtered OFDM signal. For the precoded OFDM systems, any deformation in the signal will result in a deterioration of the spectral quality. One of the most critical characteristics of the OFDM signal that is affected by the front-end electronics is the dynamic range.

The OFDM signal (both plain and precoded) exhibits high dynamic range due to its white-Gaussian-noise-like nature. The subcarriers of the OFDM signal will add together constructively (rather than destructively) most of the time due to their independent phase property. The constructive addition of the subcarriers will result on a high PAPR. We define the PAPR of one discrete OFDM symbol by

$$
\mathrm{PAPR}_{\mathrm{disc}}=\frac{\max |s[n]|^{2}}{\frac{1}{N} \sum_{n=-N_{g}}^{N_{s}}|s[n]|^{2}} .
$$

One practical visualization of the PAPR ratio is the complementary cumulative distribution function (CCDF). The CCDF curve shows the variation of the probabilities that the OFDM signal being higher than the average power with different power ratios. As the power ratio increases the probability that the OFDM signal is higher than its average power by that ratio decreases. Figure 10 shows the CCDF plots of plain versus matched and mismatched precoded discrete OFDM signals ${ }^{2}$. The figure shows that the PAPR characteristics of plain OFDM are very similar to those of precoded OFDM. For example at $\mathrm{CCDF}=8.25 \cdot 10^{-5}$ the PAPR difference between plain and precoded (both matched and mismatched) OFDM is less than $0.04 \mathrm{~dB}$ only.

The PAPR of the discrete OFDM symbol is not necessarily the same as the PAPR of the continuous OFDM symbol. We define the PAPR of the continuous OFDM symbol as

$$
\operatorname{PAPR}_{\text {cont }}=\frac{\max |s(t)|^{2}}{\frac{1}{T} \int_{t=-T_{g}}^{T_{s}}|s(t)|^{2} d t} .
$$

In fact, the PAPR of the continuous OFDM symbol may be higher than that of the discrete OFDM symbol as the comparison between Figure 10 and 11 shows. The regrowth [24] of the PAPR ratio comes as a result of the discrete to continuous conversion of the OFDM signal. Therefore, the regrowth depends on the choice of the construction and antiimaging filters at the DAC level. Figure 11 illustrates that the CCDF increases in the case of continuous OFDM signal ${ }^{3}$ compared to that of discrete OFDM signal. As an example, at PAPR of $10 \mathrm{~dB}$ the discrete OFDM systems have CCDF of almost $5 \cdot 10^{-5}$ while at the same PAPR continuous OFDM systems have CCDF of almost $10^{-3}$.

Moreover, the analog conversion of the discrete OFDM symbol does not cause a significant change between the CCDF of the continuous precoded (both matched and mismatched) OFDM signal and the CCDF of the continuous plain OFDM signal as Figure 11 shows. Although, the figure reveals that for continuous OFDM signals the mismatched precoded signal has slightly closer PAPR to the plain OFDM than the matched precoded OFDM signal.

\footnotetext{
${ }^{2}$ To get the CCDF of the PAPR shown in Figure 10, we generated random OFDM symbols that fulfill the LTE standards [4]. We measured the PAPR per OFDM symbol according to (34) then we averaged the PAPR of 10000 OFDM symbols. The OFDM system operates at 600 subcarriers mode.

${ }^{3}$ To get Figure 11 we used the same procedure used to generate Figure 10 and fulfilled (35). We converted the Discrete OFDM signal continuous using DAC of clock rate $f_{\mathrm{cl}}=\frac{1024}{T s}$ and anti-imaging filter used to generate Figure 9.
} 


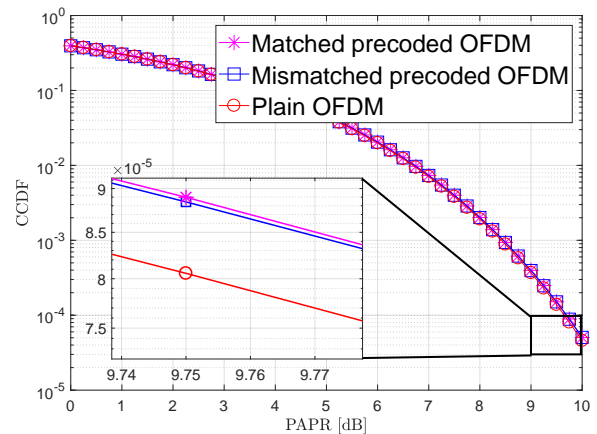

Fig. 10. CCDF characterization of discrete matched precoded, mismatched precoded and plain OFDM PAPR.

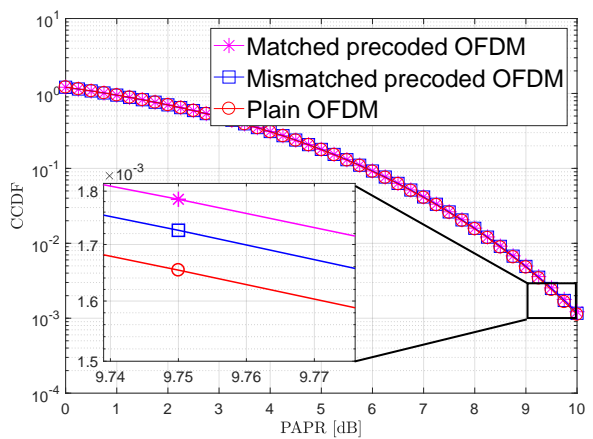

Fig. 11. CCDF characterization of continuous matched precoded, mismatched precoded and plain OFDM PAPR.

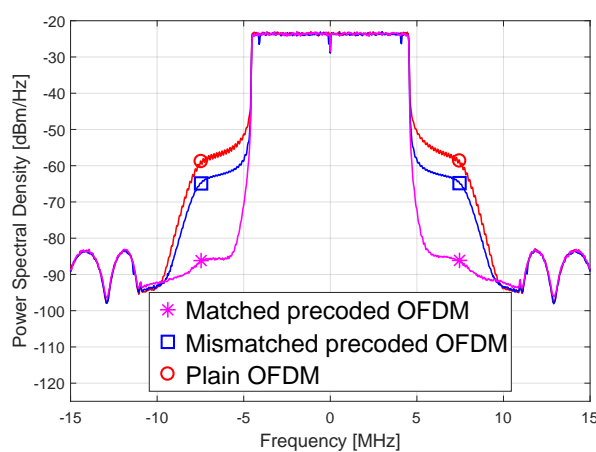

Fig. 12. The spectra of plain mismatched precoded and matched precoded OFDM signals at the output of a Rapp PA. The PA is of order 4 and back-off gain of $10 \mathrm{~dB}$.
The high dynamic range requires high cost front-end electronics especially at the PA level. PAs that support high dynamic range are more expensive than PAs that operate at lower dynamic range. Moreover, high dynamic range PAs consume more power than low dynamic range PAs and high power consumption means shorter battery life.

In practical OFDM systems, PAs neglect the high dynamic range requirements of the OFDM system. As a result, the PA will non-linearly operate over the parts of the OFDM signal with power levels higher than the linear operating region of the PA. Thus, the output amplified OFDM signal will be a distorted version of the input OFDM signal. The distortion over the OFDM signal will degrade its spectral characteristics.

To examine the influence of the PA on the spectral characteristics of the OFDM signal (both precoded and plain), we evaluated a Rapp model PA [25] with order 4 and back-off gain of $10 \mathrm{~dB}$. Using the PA we amplified a plain, mismatched precoded and matched precoded OFDM signals. Figure 12 shows the three practical spectra ${ }^{4}$ at the output of the PA. As the figure shows, the PA degrades the spectral performance of the three OFDM signals especially that of the mismatched precoded signal. On the other hand, the amplified matched precoded OFDM signal keeps a spectral improvement of at least $30 \mathrm{~dB}$ from the plain amplified OFDM. It is noteworthy to emphasize that this suppression is a result for this specific setup and it can change (improve or deteriorate) due to different design characteristics like the precoder characteristics (location of nulls), the anti-imaging filter characteristics and the PA characteristics.

\section{Performance measures of the Practical PRECODER}

As we have seen from the spectral analysis of the precoded OFDM systems, the frequency nulling precoder (especially the matched precoder) is indeed able to suppress the OOB emission. But how to check the adequacy of spectral precoding with the communications systems performance measures? In this section, we study the compliance of the matched precoding

\footnotetext{
${ }^{4}$ To estimate the practical spectrum, we ran a Welch periodogram over infinitely long OFDM signal. We generate the signal using the same setup used in Figure 11 followed by a Rapp model PA. The periodogram is Hanning windowed and uses a DFT size of 1024 and 1/8 overlapping ratio.
}

approach with different performance measures. We examine the performance of the spectral precoder at the transmitter's side of the communications link as well as at the receiver side.

At the receiver side we consider one of two regimes. In a first regime we assume that the receiver is not aware of the spectral precoding done by the transmitter. Therefore, the receiver designed is similar to the one designed for plain OFDM systems.

In a second regime we assume that the receiver is aware of the spectral precoding performed by the transmitter. Such awareness can be accomplished through a standardization of the precoding or through higher layer signaling. In this case, the receiver knows the precoder, $\mathbf{G}$, and can use an iterative algorithm to recover the original data vector $\mathbf{d}_{i}$ from the received precoded data vector [6]

$$
\mathbf{r}_{i}=\mathbf{H}_{i} \overline{\mathbf{d}}_{i}+\mathbf{n}_{i},
$$

where $\mathbf{H}_{i}$ is a $K \times K$ diagonal matrix with entries representing the channel attenuation and $\mathbf{n}_{i}$ is a $K \times 1$ vector of AWGN with zero mean and variance $\sigma^{2}$. The receiver is represented in Figure 13.

Equalization of the received precoded data vector $\mathbf{r}_{i}$ of the $i^{\text {th }}$ received OFDM symbol gives the estimated precoded data vector $\hat{\mathbf{r}}_{i} . \hat{\mathbf{r}}_{i}$ is the estimate vector of $\overline{\mathbf{d}}_{i}$. From (30), we can redefine $\overline{\mathbf{d}}_{i}$ as

$$
\overline{\mathbf{d}}_{i}=\mathbf{G d}_{i}=\mathbf{d}_{i}-\mathbf{w}_{i} .
$$

where $\mathbf{w}_{i}=(\mathbf{I}-\mathbf{G}) \mathbf{d}_{i}$, is the least square error vector between $\mathbf{d}_{i}$ and $\overline{\mathbf{d}}_{i}$ [7]. Since the receiver is of knowledge of precoding, the receiver is able to generate estimates of the errors $\mathbf{w}_{i}^{j-1}$ and add the estimated error vector to the estimated precoded data vector, $\hat{\mathbf{r}}_{i}^{j}$, to give the estimates, $\hat{\mathbf{d}}_{i}^{j}$, of the original data vector $\mathbf{d}_{i}$. Then this process is iterated. For the first iteration, i.e. $j=1, \hat{\mathbf{d}}_{i}^{1}=\hat{\mathbf{r}}_{i}$ and $\mathbf{w}_{i}^{0}=0$.

Note that the other receiver's operations such as equalization, frequency and time synchronization and carrier frequency offset (CFO) estimation remain similar to that of the conventional OFDM receiver. As we will see, the knowledge of precoding at the receiver improves the reception performance.

At the transmitter side the performance measurements include: the ACLR, the EVM and the precoder's computational 


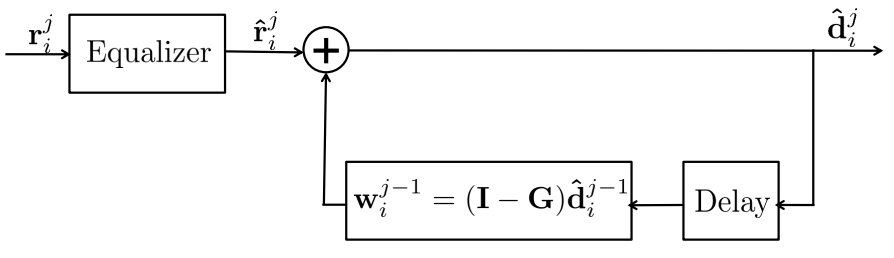

Fig. 13. Spectrally precoded OFDM iterative receiver from [6].

complexity. We discuss these measurements in the following subsections.

\section{A. ACLR}

One way to quantify the performance of the frequency nulling precoder, is to examine its compliance with the ACLR measurement. 3GPP introduces ACLR as a ratio measure of the signal power within the assigned channel bandwidth of the communications system to the OOB emission power leaking into the adjacent channels [20], [4]. For LTE, ACLR is defined as

$$
\mathrm{ACLR}=\frac{\int_{f=-\mathrm{BW} / 2}^{\mathrm{BW} / 2} \rho(f) d f}{\int_{f=-3 \mathrm{BW} / 2}^{-\mathrm{BW} / 2} \rho(f) d f+\int_{f=\mathrm{BW} / 2}^{3 \mathrm{BW} / 2} \rho(f) d f}
$$

where $\mathrm{BW}$ is the bandwidth dedicated for the communications system(including the $10 \%$ guard interval specified by LTE). In (38), $\rho(f)$ can be $\tilde{P}(f), \tilde{P}_{p}(f), P(f), P_{h}(f)$ or $P_{p}(f)$. LTE specifies that the ACLR for the different OFDM operating modes should be greater than $45 \mathrm{~dB}$ [4]. The solid lines of Figure 14 show the ACLR for plain practical OFDM systems, the ACLR for practical OFDM systems precoded with mismatched precoder, and the ACLR for practical OFDM systems precoded with matched precoder. We measure the ACLR at the input of the PA (i.e. right after the anti-imaging LPF). The systems operate in different LTE bandwidth modes. We construct the three OFDM systems using a DAC operating at a construction clock frequency $f_{\mathrm{cl}}=\frac{1024}{T_{\mathrm{s}}}$. The analog frontend filters the OFDM signal with the anti-imaging LPF we presented in Figure 9.

While the plain OFDM systems don't satisfy the ACLR requirements, the two precoded OFDM systems indeed satisfy the ACLR requirements.

Moreover, we notice that the ACLR improvement increases as the number of subcarriers in the OFDM system increases. We relate this observation to the LPF design characteristics. Since the LPF keeps a fixed cutoff frequency for the different OFDM modes, as the number of subcarriers occupied increases the BW dedicated for the OFDM system increases which means that the numerator in (38) increases. Contrarily, the denominator in (38) doesn't change due to the LPF bandpass performance. As a result the ACLR will improve as the number of subcarriers increases. We also notice that the matched precoder outperforms the mismatched precoder especially in high subcarriers number modes.

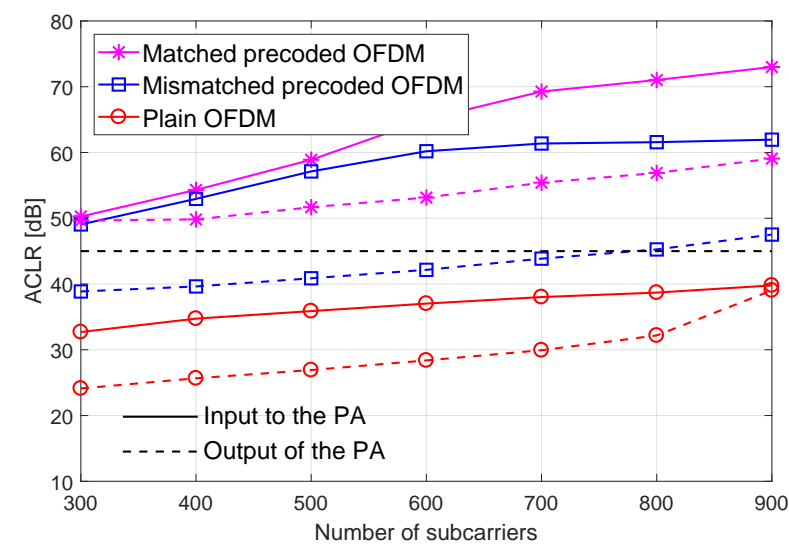

Fig. 14. ACLR measure of three OFDM systems with different number of subcarriers. We measure the ACLR of practical plain OFDM signal, practical mismatched precoded OFDM signal and practical matched precoded OFDM signal. The systems use a DAC that operates at construction clock frequency $f=\frac{1024}{T s}$ and the anti-imaging filters defined in Figure 9. The horizontal dashed line represents the ACLR LTE requirement.

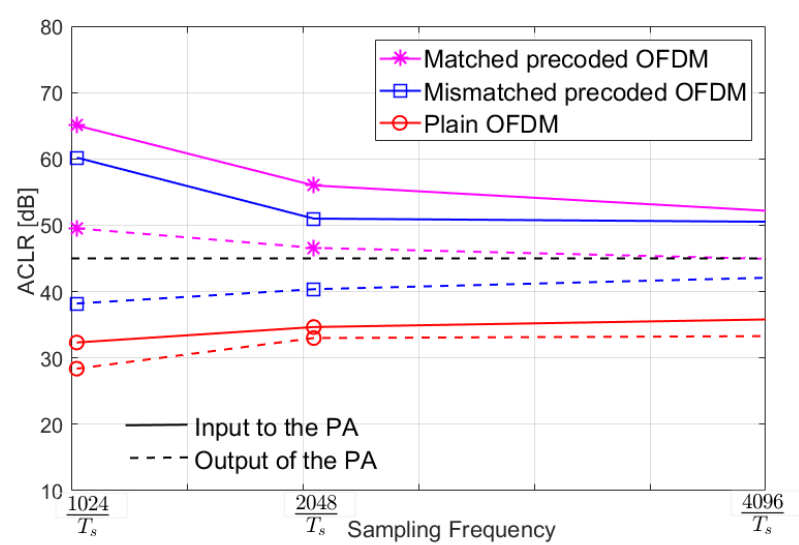

Fig. 15. ACLR measure of three OFDM systems operating at 600 subcarriers LTE mode. We measure the ACLR of practical plain OFDM signal, practical mismatched precoded OFDM signal and practical matched precoded OFDM signal. The systems use a DAC that operates at different DAC construction clock frequencies and the anti-imaging filter defined in Figure 9. The horizontal dashed line represents the ACLR LTE requirement.

The dashed lines in Figure 14 represent the ACLR for plain, mismatched precoded and matched precoded practical OFDM signals at the output of the PA. As expected from Figure 12, the spectral skirt that appears due to the non linear amplification of the OFDM signal will degrade the ACLR. While the ACLR of the plain and mismatched precoded OFDM signals at the output of the PA do not satisfy the LTE ACLR requirement, the matched precoded signal keeps satisfying the LTE requirement.

As a conclusion, an OFDM system precoded with a matched precoder and with a $7^{\text {th }}$ order Chebyshev II filter can fulfill the LTE requirements despite the PA nonlinearities. This means that the matched spectral precoding relaxes the analog filter requirements (since $7^{\text {th }}$ order filter is enough to full the ACLR requirement) and consequently decreases the complexity cost of the electronic design. 


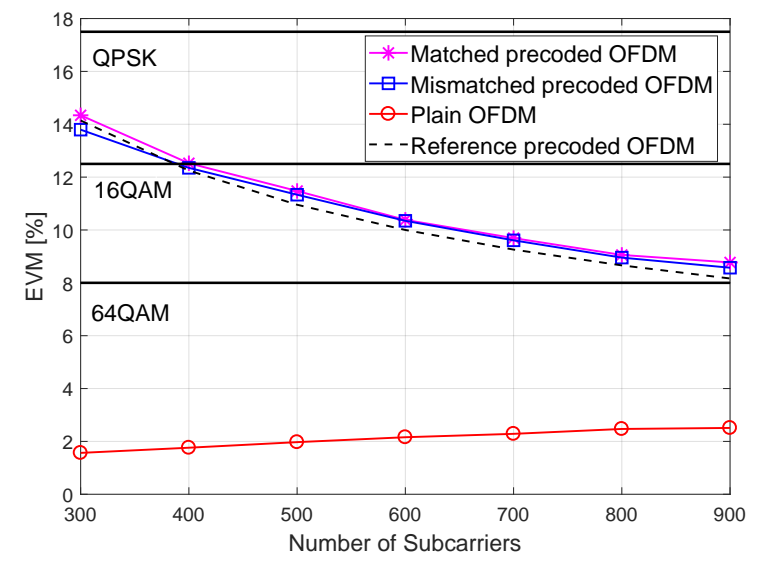

Fig. 16. Variation of the EVM performance with the number of subcarriers of practical matched precoded, mismatched precoded and plain OFDM signals at the input of the PA. We consider that spectral precoding is not included in the standards. Therefore, the receiver is not aware of spectral precoding.

Figure 15 shows the ACLR performance at different construction clock rates. It shows the ACLR for a practical OFDM system precoded with a matched precoder and the ACLR for a practical OFDM system precoded with a mismatched precoder compared to the ACLR for a plain practical OFDM system. The three OFDM systems are operating in the LTE 600 subcarriers mode. In this figure the DAC constructs the OFDM signals using construction frequency clocks $f_{\mathrm{cl}}=\frac{1024}{T_{s}}$, $\frac{2048}{T_{s}}, \frac{4096}{T_{s}}$. Again, the analog front-end filters the OFDM signal with the anti-imaging LPFs we presented in Figure 9 and amplify the OFDM signal using the PA designed for Figure 12. The solid lines show the ACLR performance of the OFDM signals at the output of the anti-imaging filter. For the plain filtered OFDM signal, the ACLR improves slightly as the construction clock frequency increases. This is expected since the construction frequency will force the filtered images away from the adjacent channel. Counterintuitively, for the precoded filtered OFDM systems we may improve the ACLR even when the DAC construction frequency decreases as Figure 15 shows. Since for spectrally precoded systems, the amount of OOB emission suppressed is more dependent on the locations of the nulling frequencies rather than the construction clock rate, therefore, if we wisely choose the nulling frequencies of the spectral precoder we can decrease the construction clock rate but still improve the ACLR!

The dashed-lines represent the ACLR of the three OFDM signals at the output of the PA. As we have shown in Figure 14, the distortion results from the PA degrades the ACLR of the three systems. Yet, regardless the PA distortion the matched precoder keeps fulfilling the ACLR requirements of LTE.

\section{B. $E V M$}

The EVM is a percentage measure considered by 3GPP to quantify how much the implemented OFDM signal is close to the reference OFDM signal. LTE defines the EVM as

$$
\mathrm{EVM}=\sqrt{\frac{E\left\|\check{\mathbf{d}}_{i}-\mathbf{d}_{i}\right\|^{2}}{E\left\|\mathbf{d}_{i}\right\|^{2}}}
$$

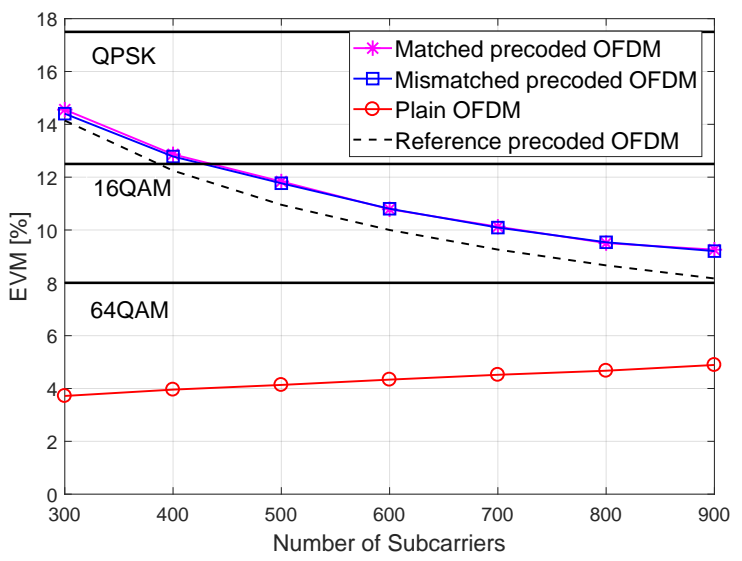

Fig. 17. Variation of the EVM performance with the number of subcarriers of practical matched precoded, mismatched precoded and plain OFDM signals at the output of the PA. We consider that spectral precoding is not included in the standards. Therefore, the receiver is not aware of spectral precoding.

In (39), $\check{\mathbf{d}}_{i}$ is the data vector received by a receiver connected directly after the OFDM transmitter (i.e. the OFDM signal transmitted doesn't pass through any communications channel). As [7] shows, for precoded reference OFDM system, $\check{\mathbf{d}}_{i}=\overline{\mathbf{d}}_{i}$. Consequently (39) becomes

$$
\mathrm{EVM}=\sqrt{\frac{M}{K}},
$$

where $M$ as we mentioned earlier is the number of frequency nulls. This means that for precoded reference OFDM systems, the EVM increases with the number of nulls and decreases with the number of subcarriers.

For practical OFDM systems, the EVM will degrade. While the anti-imaging LPF (in our analysis we used the LPF also used in Figure 9) will slightly degrade the EVM of the precoded OFDM systems, the PA will degrade the EVM significantly. Actually,

$$
\mathrm{EVM}>\sqrt{\frac{M}{K}} .
$$

In Figure 16 we show the EVM for three practical OFDM signals: matched precoded, mismatched precoded and plain at the output of the anti-imaging filter. The figure shows the variation of the EVM performance with the number of subcarriers. The EVM measures of the three systems are compared with the EVM of a precoded reference OFDM system that fulfills (40). As expected from (40), for the precoded systems, increasing the number of subcarriers will decrease the EVM.

The three solid lines in the figure represent the maximum EVM allowed by LTE for QPSK, 16QAM and 64QAM mapping schemes respectively. We notice for QPSK modulation schemes precoding does not violate the EVM specifications. For 16QAM schemes precoding violates the EVM specifications for OFDM modes with low number of subcarriers ( $K<400)$. While for 64QAM schemes precoding violates the EVM specifications for most of the OFDM modes.

The PA distortion will degrade the EVM performance even more. Figure 17 shows the variation of the EVM with the 


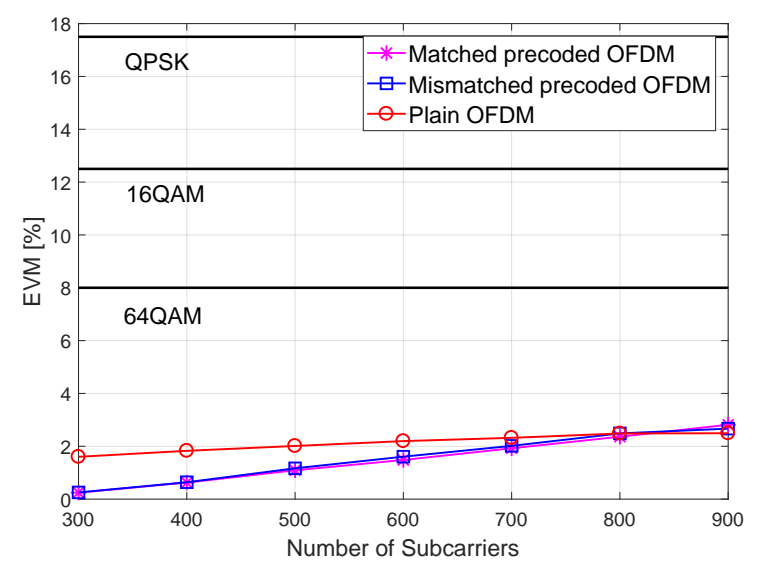

Fig. 18. Variation of the EVM performance with the number of subcarriers of practical matched precoded, mismatched precoded and plain OFDM signals at the input of the PA. We consider that spectral precoding is included in the standards. Therefore, the receiver is aware of spectral precoding.

number of subcarriers of the three signals used in Figure 16 but at the output of the PA. One noteworthy observation is the fact that the total output EVM shown in Figure 17 is not the result of addition of the EVMs measured at the consequent stages of the OFDM transmitter. Unfortunately, It is clear that the EVM levels of the precoded amplified output signals violate the LTE acceptable EVM level.

Although, the spectral precoder noticeably degrades the EVM performance and violates the standards specifications, it is noteworthy to mention that these EVM specifications assume the use of the typical OFDM receiver which is not aware of the spectral precoding process. If the spectral precoding becomes a part of the standards then the EVM performance of the spectrally precoded systems will improve. In case of standardization of spectral precoding the definition of the EVM becomes

$$
\mathrm{EVM}=\sqrt{\frac{E\left\|\check{\mathbf{d}}_{i}-\overline{\mathbf{d}}_{i}\right\|^{2}}{E\left\|\overline{\mathbf{d}}_{i}\right\|^{2}}}
$$

and therefore, the EVM performance improves as shown in Figure 18 and 19. While Figure 18 shows the EVM of the OFDM signal at the input of the PA, Figure 19 shows the EVM of the OFDM signal at the output of the PA. Although we notice a degradation in the EVM of the OFDM signal at the output of the PA, yet despite the degradation, the EVM fulfills the LTE requirement. This means that the standardization of the spectral precoding saves the EVM performance from being violated.

\section{Complexity}

Besides ACLR and EVM, implementation complexity is another measurement that qualifies the transmitter of the spectrally precoded OFDM. We can classify the complexity into off-line and on-line complexity. In off-line complexity the system computes the precoding matrix $\mathbf{G}$ and load it in the system's memory. The off-line computations occur once prior transmission (if $K$ and $M$ change adaptively) or even

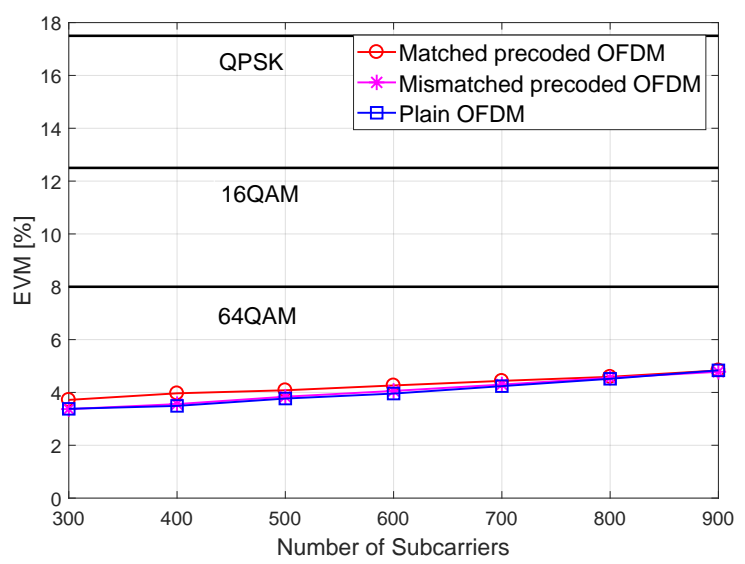

Fig. 19. Variation of the EVM performance with the number of subcarriers of practical matched precoded, mismatched precoded and plain OFDM signals at the output of the PA. We consider that spectral precoding is included in the standards. Therefore, the receiver is aware of spectral precoding.

TABLE II

COMPUTATIONAL COMPLEXITY COMPARISON BETWEEN PLAIN AND PRECODED OFDM TRANSMITTERS

\begin{tabular}{|c|c|c|}
\hline & $\begin{array}{c}\text { Number of } \\
\text { multiplications } \\
\text { per OFDM symbol }\end{array}$ & $K=600, M=6, N_{s}=1024$ \\
\hline Plain OFDM & $N_{s} \log _{2}\left(N_{s}\right)$ & $\begin{array}{c}\text { Increase in } \\
\text { computational } \\
\text { complexity }\end{array}$ \\
\hline $\begin{array}{c}\text { Precoded OFDM } \\
\text { original }\end{array}$ & $K^{2}+N_{s} \log _{2}\left(N_{s}\right)$ & $\frac{K^{2}}{N_{s} \log _{2}\left(N_{s}\right)}=3516 \%$ \\
\hline $\begin{array}{c}\text { Precoded OFDM } \\
\text { according to } \\
\text { Figure 20 }\end{array}$ & $2 M K+N_{s} \log _{2}\left(N_{s}\right)$ & $\frac{2 M K}{N_{s} \log _{2}\left(N_{s}\right)}=70 \%$ \\
\hline
\end{tabular}

stored permanently in the memory (if $K$ and $M$ don't change adaptively). On the other hand, the on-line complexity represents the number of computational processes required during transmission. on-line, the transmitter precodes the original data vector $\mathbf{d}_{i}$ into $\overline{\mathbf{d}}_{i}$ by multiplying $\mathbf{d}_{i}$ with $\mathbf{G}$. Here, we focus on the on-line complexity.

At first look, the precoding matrices $\mathbf{G}$ and $\tilde{\mathbf{G}}$ are $K \times K$ matrices. This means that the computational complexity of precoding will grow in order $O\left(K^{2}\right)$. However, elegant implementation of the precoder will significantly decrease the computation complexity [26]. If we redefine the matched precoder $\mathbf{G}$ as

$$
\mathbf{G}=\mathbf{I}-\mathbf{V B}
$$

where $\mathbf{V}=\mathbf{B}^{H}\left(\mathbf{B B}^{H}\right)^{-1}$ is a $K \times M$ matrix and $\mathbf{B}$ is an $M \times K$ matrix, then we can perform the precoding operation in (19) in two steps as illustrated in Figure 20. Firstly, we compute

$$
\overline{\mathbf{d}}_{i}^{\prime}=\mathbf{B d}_{i}
$$

a step that costs the system $M K$ complex multiplications for each OFDM symbol. Secondly, we compute

$$
\overline{\mathbf{d}}_{i}=\mathbf{d}_{i}-\mathbf{V} \overline{\mathbf{d}}_{i}^{\prime}
$$

which also costs the system $M K$ complex multiplications for each OFDM symbol. Consequently, if we store $\mathbf{V}$ and 
B in the system's memory instead of $\mathbf{G}$ then precoding will cost the system $2 M K$ multiplications per OFDM symbol. On the other hand, the IDFT process will cost the system $N_{s} \log _{2}\left(N_{s}\right)$ complex multiplications assuming that the system implements the IDFT block using fast Fourier transform (FFT) algorithm. Therefore, the total multiplication complexity of the system will be $2 M K+N_{s} \log _{2}\left(N_{s}\right)$. Taking into consideration that $M<<K$, the implementation of the precoder using the discussed two stages significantly decreases the computational complexity. Table II summarizes the comparison between the implementation complexities at the transmitter of plain OFDM, precoded OFDM implemented according to the original way and precoded OFDM implemented according to the technique introduced in Figure 20.

On the other hand, if we consider the iterative decoder at the receiver side then the complexity will grow linearly with the number of iterations. As Figure 13 shows each iteration within the iterative decoder requires multiplication of the iterated data vector $\hat{\mathbf{d}}_{i}^{j-1}$ with $\mathbf{I}-\mathbf{G}$. As Figure 20 shows multiplying with $\mathbf{I}-\mathbf{G}$ requires $2 M K$ complex multiplications. Therefore the computation complexity of the receiver will increase by $2 J K M$ complex multiplications where $J$ is the total number of iterations.

Besides complexity the performance measurements at the receiver include: the in-band interference measure and the Bit Error Rate (BER) performance. We study these measurements in the following subsection.

\section{In-band Interference and BER performance}

The frequency nulling spectral precoding comes with a price [22]. Since the precoder linearly combines the original data symbols, $\mathbf{d}_{i}$, the precoded data symbols become correlated, i.e. $E\left\{\overline{\mathbf{d}}_{i} \overline{\mathbf{d}}_{i}^{H}\right\} \neq \mathbf{I}$. This correlation between the precoded data symbols appears in the form of in-band interference. We can derive this in-band interference in case the communications link uses the first regime receiver. i.e. a receiver that is not aware of spectral precoding.

Due to the mathematical similarity between the mismatched precoder, $\tilde{\mathbf{G}}$ and the matched precoder, $\mathbf{G}$, we naturally adopt the analysis in [22] for the matched precoding technique. Similar to [22], the in-band interference due to the discretetime spectral precoding is not equally distributed over the different subcarriers of the OFDM symbol,

$$
\begin{aligned}
D & =\operatorname{diag}\left(E\left\{\left(\mathbf{d}_{i}-\overline{\mathbf{d}}_{i}\right)\left(\mathbf{d}_{i}-\overline{\mathbf{d}}_{i}\right)^{H}\right\}\right) \\
& =\operatorname{diag}\left(E\left\{\left(\boldsymbol{\Theta} \mathbf{d}_{i}\right)\left(\boldsymbol{\Theta} \mathbf{d}_{i}\right)^{H}\right\}\right) \\
& =\operatorname{diag}\left(\boldsymbol{\Theta} \boldsymbol{\Theta}^{H}\right)=\operatorname{diag}(\boldsymbol{\Theta}),
\end{aligned}
$$

where $\mathbf{\Theta}=\mathbf{I}-\mathbf{G}=\mathbf{A}^{H}\left(\mathbf{A} \mathbf{A}^{H}\right)^{-1} \mathbf{A}$, is the projection matrix that projects $\mathbf{d}_{i}$ onto the null space, $\mathcal{N}(\mathbf{A})$, of $\mathbf{A}$.

From $\Theta$ we can say that the total in-band interference due to discrete-time precoding over one OFDM symbol is

$$
D_{\text {total }}=\operatorname{trace}\{\boldsymbol{\Theta}\}=\operatorname{rank}\{\mathbf{A}\}=M=\left|\mathcal{S}_{1}\right| .
$$
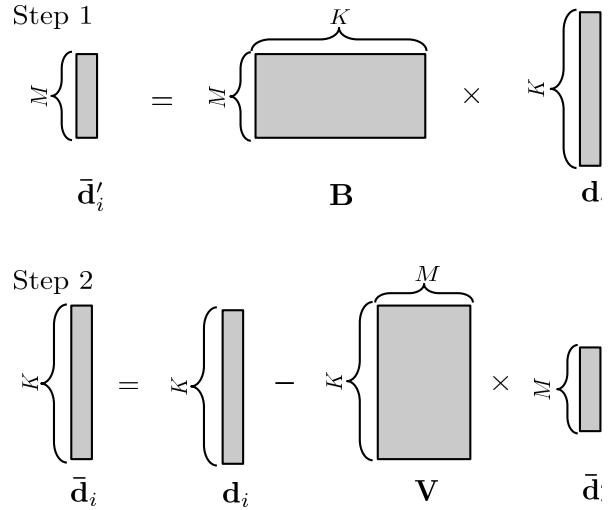

Fig. 20. Illustration of the computational complexity of the spectral precoder. Each of the two steps requires $M K$ multiplications per OFDM symbol.

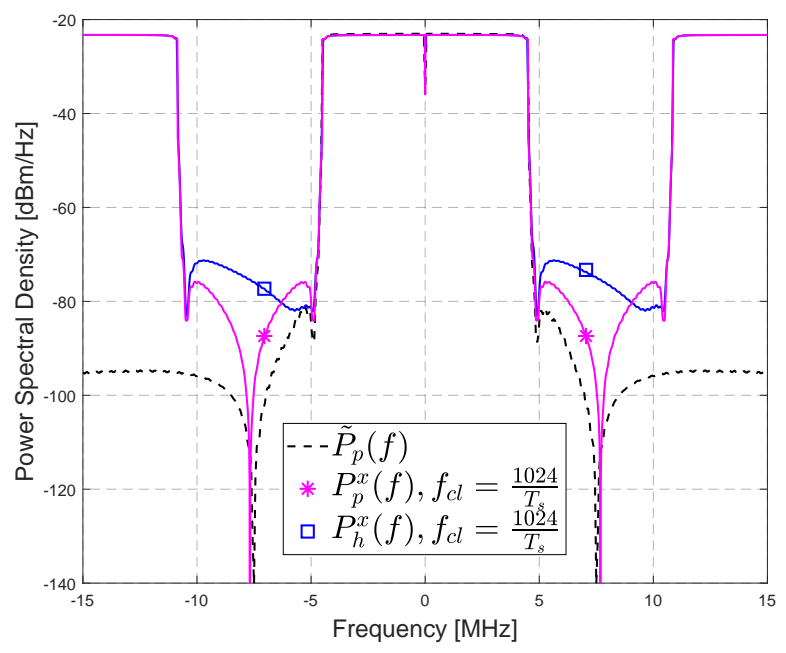

Fig. 21. Spectrum of a practical OFDM signal precoded with a mismatched precoder $\left(P_{h}^{x}(f)\right)$ versus the spectrum of a practical OFDM signal precoded with a matched precoder $\left(P_{p}^{x}(f)\right.$ compared to the spectrum of a precoded reference OFDM signal $\left(\tilde{P}_{p}(f)\right)$. We used the same setup as Figure 7 but the precoder introduces 6 nulls rather than 8 at $f_{m} \in \hat{\mathcal{S}}$.

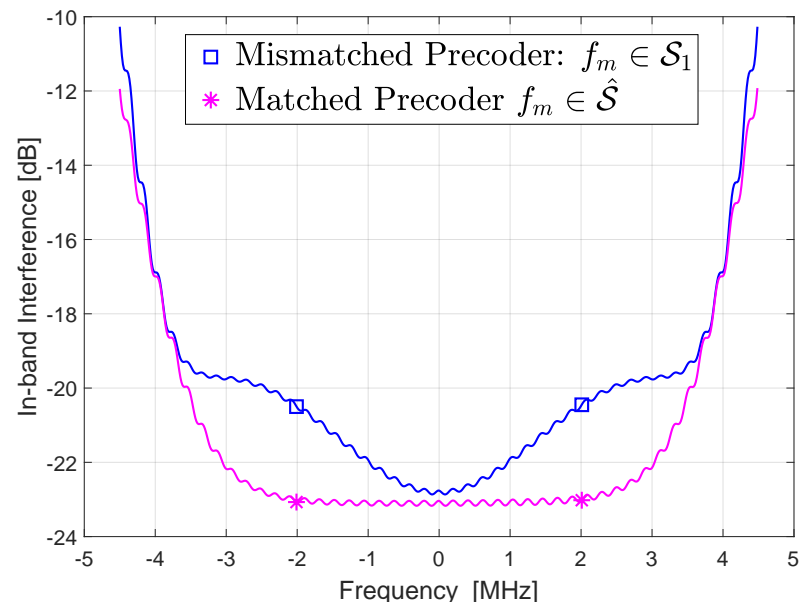

Fig. 22. The in-band interference over the subcarriers of two practical OFDM systems. One system is precoded with a matched precoder of reduced number of nulls $(\hat{\mathcal{S}})$. The other system is precoded with a mismatched precoder of regular number of nulls $\left(\mathcal{S}_{1}\right)$. The system uses the same setup as Figure 7. 
In words, the total in-band interference (relative to the energy of the OFDM symbol) due to spectral precoding is equal to the total number of nulls.

For the practical OFDM model, and due to the circularity of the constructed signal's spectrum around $f_{\mathrm{cl}}$, a null placed at $f_{m}=-\frac{f_{\mathrm{cl}}}{2}$ is equivalent to a null placed at $f_{m}=\frac{f_{\mathrm{cl}}}{2}$. This means that if we design the matched precoder to null the spectrum close to $\frac{f_{\mathrm{cl}}}{2}$ we can save a pair of nulls (due to the fact that each two nulls are coupled together). Yet, we still get the same amount of OOB emission suppression. For example we can obtain $P_{p}^{x}(f)$ as in Figure 7 if we define $f_{m} \in$ $\hat{\mathcal{S}}=\{+7.68,+7.67, \pm 4.85, \pm 4.86\}$ i.e. the cardinality of $\hat{\mathcal{S}}$, $|\hat{\mathcal{S}}|=6$ rather than 8 . Generally, we can say that $|\hat{\mathcal{S}}|=\left|\mathcal{S}_{1}\right|-2$.

In contrast, we cannot decrease the number of nulls for the mismatched precoder while maintaining the suppression level of the OOB emission. The mismatched precoder assumes that the signal is originally continuous and therefore the spectrum is not circular. If we discard one of the pairs in the mismatched precoder implementation the spectrum of the OFDM signal will be asymmetric. We show that in Figure 21. Figure 22 compares the in-band interference over the subcarriers of two OFDM systems precoded with matched versus mismatched precoders.

It is noteworthy to mention that if the communications system uses the second regime receiver (i.e. the iterative receiver described in Figure 13 then the in-band residual interference is smaller than (46) and (47).

The in-band interference due to spectral precoding also affects the BER performance. Figure 23 shows the BER performance of matched precoded versus mismatched precoded OFDM compared to plain OFDM. The systems transmit the signals in an AWGN channel. Again we use the precoders that generate the OFDM signals with spectra shown in Figure 7 (The system is operating at 600 subcarriers mode). The figure shows the performance of three constellation-size communication systems (QPSK, 16-QAM and 64-QAM). We included a turbo coded BER performance specified by the LTE standards with code rate $1 / 2$. Table III concludes the simulation setup required to generate Figure 23. While the solid lines show the performance of the blind receiver that has no knowledge of spectral precoding at the transmitter side. The dashed lines show the performance of the iterative receiver we studied in section $\mathrm{V}$. The receiver we used is an 8-iteration receiver. As Figure 23 shows the iterative receiver has better BER than the blind receiver especially for the high constellation sets.

\section{VI, CONCLUSION}

In this paper, we studied the implementation architecture of the spectrally precoded OFDM transmitter. We introduced a novel frequency nulling spectral precoder that operates in conjunction with the practical OFDM modulators. We showed that spectral precoding helps simplifying the design of the analog anti-imaging filters of the OFDM transmitters. we also show that spectral precoding does not have a significant drawback on the PAPR of the OFDM signal. Moreover, we discussed the influence of the PA on the performance of the spectral
TABLE III

SimULATION SETUP FOR FigURE 23

\begin{tabular}{|c|c|}
\hline Symbol length: $T_{s}$ & $\frac{1}{15} \mathrm{~ms}$ \\
\hline CP length: $T_{g}$ & $\approx 4.7 \mu \mathrm{s}$ \\
\hline Number of subcarriers: $K$ & 600 \\
\hline DFT block size: $N_{s}$ & 1024 \\
\hline Number of nulls: $M$ & $\begin{array}{c}6 \text { for matched precoder } \\
8 \text { for mismatched precoder }\end{array}$ \\
\hline Channel & AWGN \\
\hline LTE coding scheme & Turbo code $=1 / 2$ \\
\hline $\begin{array}{c}\text { Number of decoding iterations } \\
\text { (iterative receiver only) }\end{array}$ & 8 \\
\hline
\end{tabular}

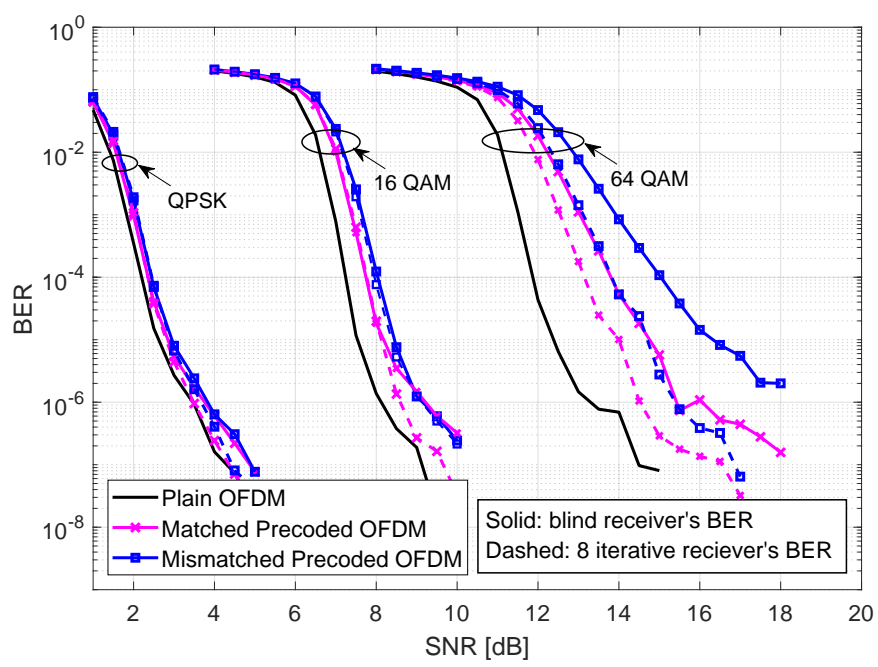

Fig. 23. coded BER performance of matched precoded OFDM system versus mismatched precoded OFDM system compared to plain OFDM system. The system uses LTE Turbo codes with $1 / 2$ coding rate and we use the same precoding setup of Figure 7. The figure shows the BER performance of three constellation sizes: QPSK, 16 QAM and 64 QAM. The solid lines represent a blind receiver BER performance while the dashed lines represent an 8 iterative receiver BER performance.

precoding. We noticed that the inter-modulation caused by the PA degrades the spectral precoding suppression performance of the mismatched precoders. Furthermore, we compared the compliance of the spectrally precoded OFDM systems with the 3GPP measures. We analyzed the computation complexity of the precoder as well. At the receiver side we studied the inband interference caused by the spectral precoding and BER performances where we found that our novel spectral precoder outperforms the traditional spectral precoder.

\section{REFERENCES}

[1] $5 G N R$ (Release 14), 3GPP TSG RAN meeting Std., Mar. 2016 [Online]. Available: http://www.3gpp.org/

[2] Evolved Universal Terrestrial Radio Access (E-UTRA); Physical Channels and Modulation (Release 8), 3GPP TSG RAN TS 36.211, v8.5.0., Dec. 2008. [Online]. Available: http://www.3gpp.org/

[3] I. Cosovic, S. Brandes, and M. Schnell, "Subcarrier weighting: a method for sidelobe suppression in OFDM systems," IEEE Communications Letters, vol. 10, no. 6, pp. 444-446, June 2006.

[4] Base Station (BS) Radio Transmission and Reception (Release 8), 3GPP TSG RAN TS 36.104, v8.4.0., Dec. 2008. [Online]. Available: http://www.3gpp.org/

[5] C. D. Chung, "Spectrally Precoded OFDM," IEEE Transactions on Communications, vol. 54, no. 12, pp. 2173-2185, Dec 2006. 
TABLE IV

SUMMARY OF SYMBOLS

\begin{tabular}{|c|c|c|c|c|c|c|c|c|c|}
\hline symbol & $T_{s}$ & $T_{g}$ & $T$ & $K$ & $N_{s}$ & $N_{g}$ & $N$ & $\mathbf{d}_{i}$ & $\overline{\mathbf{d}}_{i}$ \\
\hline description & $\begin{array}{c}\text { OFDM symbol } \\
\text { length }\end{array}$ & $\begin{array}{c}\text { CP } \\
\text { length }\end{array}$ & $T_{s}+T_{g}$ & $\begin{array}{c}\text { Number of } \\
\text { subcarriers }\end{array}$ & DFT size & $\begin{array}{c}\text { Number of } \\
\text { samples in CP }\end{array}$ & $N_{s}+N_{g}$ & $\begin{array}{c}\text { Original } \\
\text { data vector }\end{array}$ & $\begin{array}{c}\text { Precoded } \\
\text { data vector }\end{array}$ \\
\hline
\end{tabular}

\begin{tabular}{|c|c|c|c|c|c|c|c|c|c|}
\hline symbol & $f_{\mathrm{cl}}$ & $f_{c}$ & $f_{m}$ & $M$ & $j$ & $J$ & $\mathbf{w}_{i}$ & $\check{\mathbf{d}}_{i}$ & $\mathbf{r}_{i}$ \\
\hline description & $\begin{array}{c}\text { DAC } \\
\text { clock }\end{array}$ & $\begin{array}{c}\text { Carrier } \\
\text { frequency }\end{array}$ & $\begin{array}{c}m^{\text {th }} \text { nulling } \\
\text { frequency }\end{array}$ & $\begin{array}{c}\text { Number of } \\
\text { nulls }\end{array}$ & $\begin{array}{c}\text { Decoder } \\
\text { iteration index }\end{array}$ & $\begin{array}{c}\text { Number of decoder } \\
\text { iterations }\end{array}$ & $\begin{array}{c}\text { LSE } \\
\text { vector }\end{array}$ & $\begin{array}{c}\text { Estimated } \\
\text { data vector }\end{array}$ & $\begin{array}{c}\text { Received precoded } \\
\text { data vector }\end{array}$ \\
\hline
\end{tabular}

[6] J. van de Beek and F. Berggren, " $N$-continuous OFDM," IEEE Communications Letters, vol. 13, no. 1, pp. 1-3, January 2009.

[7] — "EVM-Constrained OFDM Precoding for Reduction of Out-ofBand Emission," in Proc, IEEE Vehicular Technology Conference, Sept 2009, pp. 1-5.

[8] H. Kawasaki, M. Ohta, and K. Yamashita, " $N$-continuous symbol padding OFDM for sidelobe suppression," in Proc, IEEE International Communications Conference (ICC), June 2014, pp. 5890-5895.

[9] J. van de Beek, "Sculpting the multicarrier spectrum: a novel projection precoder," IEEE Communications Letters, vol. 13, no. 12, pp. 881-883, 2009.

[10] A. Tom, A. Sahin, and H. Arslan, "Mask compliant precoder for OFDM spectrum shaping," IEEE Communications Letters, vol. 17, no. 3, pp. 447-450, 2013.

[11] X. Zhou, G. Y. Li, and G. Sun, "Multiuser spectral precoding for OFDMbased cognitive radio systems," IEEE Journal on Selected Areas in Communications, vol. 31, no. 3, pp. 345-352, 2013.

[12] M. Ma, X. Huang, B. Jiao, and Y. J. Guo, "Optimal orthogonal precoding for power leakage suppression in DFT-based systems," IEEE Transactions on Communications, vol. 59, no. 3, pp. 844-853, 2011.

[13] R. W. Chang, "Synthesis of Band-Limited Orthogonal Signals for Multichannel Data Transmission," Bell Labs Technical Journal, vol. 45, no. 10 , pp. $1775-1796,1966$.

[14] J. A. Bingham, "Multicarrier modulation for data transmission: An idea whose time has come," IEEE Communications magazine, vol. 28, no. 5, pp. 5-14, 1990.

[15] J. F. Schmidt, S. Costas-Sanz, and R. Lopez-Valcarce, "Choose your subcarriers wisely: Active interference cancellation for cognitive OFDM," IEEE Journal on Emerging and Selected Topics in Circuits and Systems, vol. 3, no. 4, pp. 615-625, 2013.

[16] R. Xu and M. Chen, "A precoding scheme for DFT-based OFDM to suppress sidelobes," IEEE Communications Letters, vol. 13, no. 10, 2009.

[17] Y.-P. Lin and S.-M. Phoong, "OFDM transmitters: analog representation and DFT-based implementation," IEEE Transactions on Signal Processing, vol. 51, no. 9, pp. 2450-2453, 2003.

[18] D. Markert, X. Yu, H. Heimpel, and G. Fischer, "An All-Digital, SingleBit RF Transmitter for Massive MIMO," IEEE Transactions on Circuits and Systems I: Regular Papers, vol. 64, no. 3, pp. 696-704, 2017.

[19] A. V. Oppenheim and R. W. Schafer, Discrete-Time Signal Processing, 3rd ed. Pearson, 2014

[20] E. Dahlman, S. Parkvall, and J. Skold, 4G: LTE/LTE-advanced for mobile broadband. Academic press, 2013.

[21] M. Faulkner, "The effect of filtering on the performance of OFDM systems," IEEE Transactions on Vehicular Technology, vol. 49, no. 5, pp. 1877-1884, 2000.

[22] M. Mohamad, R. Nilsson, and J. v. d. Beek, "An analysis of out-of-band emission and in-band interference for precoded and classical OFDM systems," in Proc, IEEE European Wireless Conference, May 2015, pp. $1-5$.

[23] M. Mohamad, R. Nilsson, and J. van de Beek, "An SDR-based prototype of spectrally precoded OFDM," in Proc, IEEE European Conference on Networks and Communications (EuCNC), May 2017, pp. 1-6.

[24] J. Armstrong, "Peak-to-average power reduction for OFDM by repeated clipping and frequency domain filtering," Electronics letters, vol. 38, no. 5, pp. 246-247, 2002.

[25] C. Rapp, "Effects of HPA-nonlinearity on a 4-DPSK/OFDM-signal for a digital sound broadcasting signal," in Proc, ESA Second European Conference on Satellite Communications (ECSC-2), 1991, pp. 179-184.
[26] M. Mohamad, R. Nilsson, and J. van de Beek, "Minimum-EVM Ncontinuous OFDM," in Proc, IEEE International Communications Conference (ICC), May 2016, pp. 1-5.

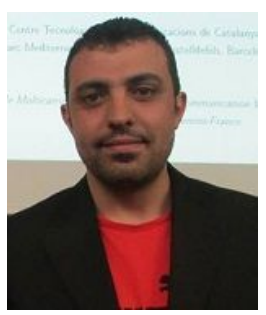

Medhat Mohamad received his B.S. degree in Electronics and Communication Engineering from Beirut Arab university (BAU), Beirut, Lebanon, in 2010, the M.Sc degree in Wireless Communication from Lund Tekniska Högskola (LTH), Lund, Sweden, in 2013 and the Lic.Eng. degree from Luleå Tekniska Universitet (LTU), Luleå, Sweden in 2016. Currently he is working towards his $\mathrm{PhD}$ degree at LTU. His research interests are physical layer for radio communications, modulations, waveform designs, software designed radio (SDR) and signal processing techniques for multi-carrier systems and dynamic access regimes.

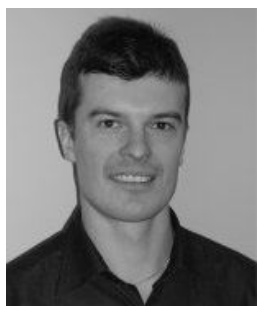

Rickard Nilsson received the M.Sc., Lic. Eng., and $\mathrm{Ph} . \mathrm{D}$. degrees from LuleåUniversity of Technology (LTU), Sweden. With Telia Research AB, Sweden, and Stanford University, USA, he developed and researched a new broadband access waveformtechnology for VDSL and contributed to its international standardization; a successful technology that enabled true broadband Internet access to many millions of homes and offices worldwide over existing telephone wires. For seven years he lived in Vienna, Austria, where he researched broadband access technologies at the Telecommunications Research Center Vienna (FTW) in close cooperation with operators, system manufacturers, and chip vendors. In Vienna he also lectured at the Technical University and co-supervised Ph.D. students. In 2010 he returned to LTU and founded a new research group with wireless communications and software radio and established new cooperation with mining and telecom industries. At LTU he continues to lecture Signal Processing and Communications courses and supervise Ph.D. students. His research interest is broad and spans from understanding both theory and practice in order to develop new applicable algorithms and methods.

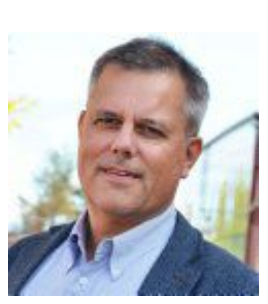

Jaap van de Beek is chaired professor of Signal Processing with LuleåUniversity of Technology, Sweden. Prior to his return to academia in 2013, he spent over two decades in industry, in telecommunications research labs with Telia Research, Nokia Networks, and for more than twelve years with Huawei Technologies. His work has mainly concentrated on the physical layer of radio access networks and, while at Huawei, he contributed to the preparation and specification the LTE standard for which he holds a number of essential patents. Prof. van de Beek has served as an editor of the IEEE Communications Letters and the IEEE ComSoc Technology News. He is an IEEE Fellow and received the IEEE ComSoc Heinrich Hertz award in 2010. His research today includes waveforms for dynamic spectrum-access regimes, radio environment mapping, and he is engaged in the development of rural regions and the improvement of Internet access, connectivity and cellular radio coverage. 